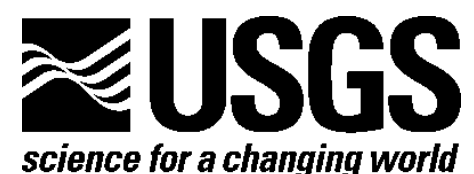

science for a changing world

\title{
Radioisotopic Data of Sediment Collected in Mobile and
}

\section{Bon Secour Bays, Alabama}

By Marci E. Marot and Christopher G. Smith

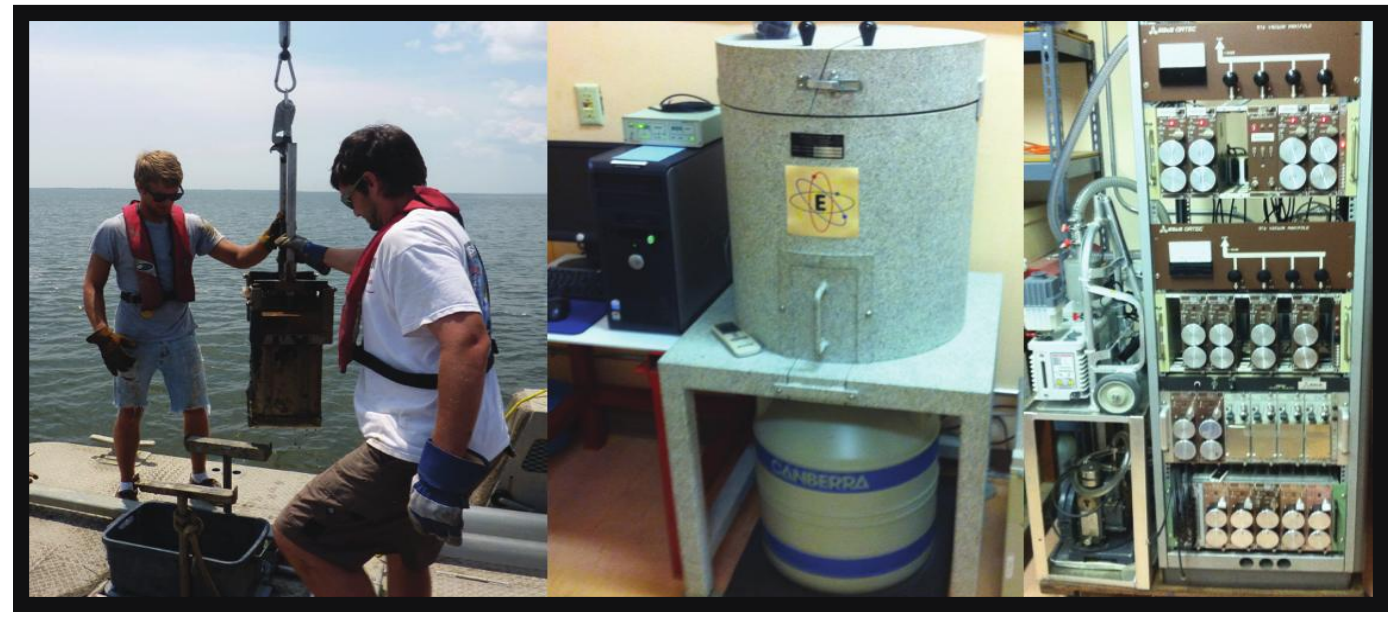

Open-File Report 2012-1172

U.S. Department of the Interior

U.S. Geological Survey 


\section{U.S. Department of the Interior \\ KEN SALAZAR, Secretary}

\section{U.S. Geological Survey \\ Marcia K. McNutt, Director}

U.S. Geological Survey, Reston, Virginia: 2012

For more information on the USGS-the Federal source for science about the Earth,

its natural and living resources, natural hazards, and the environment-visit

http://www.usgs.gov or call 1-888-ASK-USGS

For an overview of USGS information products, including maps, imagery, and publications, visit $h$ ttp://www.usgs.gov/pubprod

To order this and other USGS information products, visit $h$ ttp://store.usgs.gov

Suggested citation:

Marot, M.E., and Smith, C.G., 2012, Radioisotopic data of sediment collected in Mobile and Bon Secour Bays, Alabama: U.S. Geological Survey Open-File Report 2012-1172, 15 p.

Any use of trade, product, or firm names is for descriptive purposes only and does not imply endorsement by the U.S. Government.

Although this report is in the public domain, permission must be secured from the individual copyright owners to reproduce any copyrighted material contained within this report.

Front photographs: C. Smith and A. Atchinson collecting box cores onboard the R/V G.K. Gilbert; Gamma spectroscopy system, USGS, St. Petersburg, FL; Alpha spectroscopy system USGS, St. Petersburg, FL. 


\section{Contents}

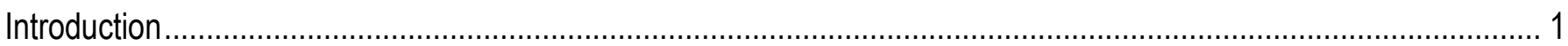

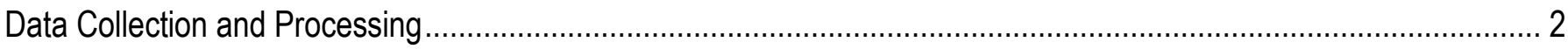

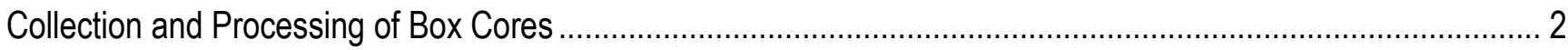

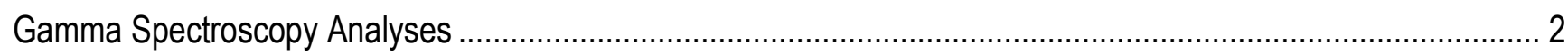

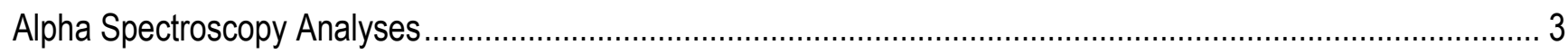

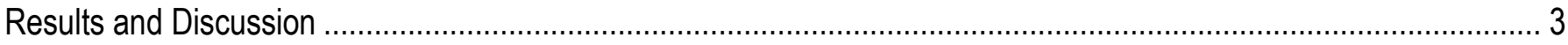

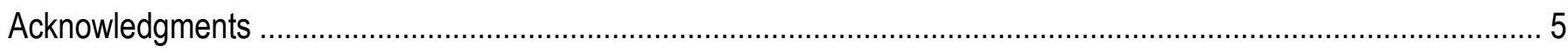

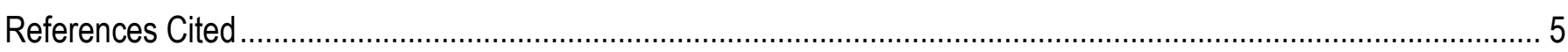

\section{Figures}

1. Site locations in Mobile and Bon Secour Bays, Alabama........................................................................

\section{Tables}

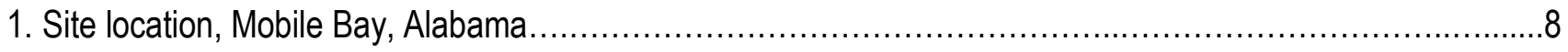

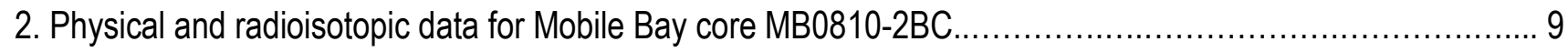

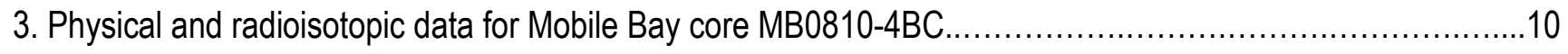

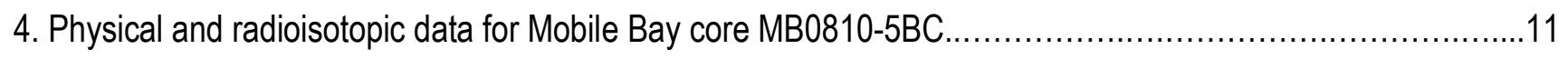

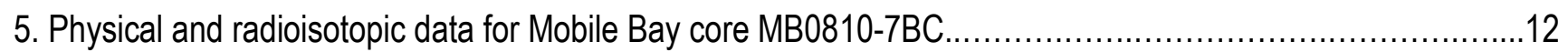

6. Physical and radioisotopic data for Mobile Bay core MB0810-8BC .................................................... 13

7. Physical and radioisotopic data for Mobile Bay core MB0810-12BC ................................................. 14

8. Physical and radioisotopic data for Mobile Bay core MB0810-20BC ............................................. 15 


\section{Conversion Factors}

\begin{tabular}{|c|c|c|}
\hline Multiply & By & To obtain \\
\hline \multicolumn{3}{|c|}{ Length } \\
\hline centimeter $(\mathrm{cm})$ & 0.3937 & inch (in.) \\
\hline \multicolumn{3}{|c|}{ Volume } \\
\hline milliliter $(\mathrm{mL})$ & 0.03382 & ounce, fluid (fl. oz) \\
\hline \multicolumn{3}{|c|}{ Mass } \\
\hline $\operatorname{gram}(\mathrm{g})$ & 0.03527 & ounce, avoirdupois (oz) \\
\hline \multicolumn{3}{|c|}{ Density } \\
\hline gram per cubic centimeter $\left(\mathrm{g} / \mathrm{cm}^{3}\right)$ & 62.4220 & pound per cubic foot $\left(\mathrm{lb} / \mathrm{ft}^{3}\right)$ \\
\hline \multicolumn{3}{|c|}{ Radioactivity } \\
\hline disintegration per minute (dpm) & 0.45 & picocurie (pCi) \\
\hline \multicolumn{3}{|c|}{ Energy } \\
\hline kiloelectronvolt (keV) & $1.60 \mathrm{E}-16$ & Joule (J) \\
\hline
\end{tabular}




\section{Radioisotopic Data of Sediment Collected in Mobile and}

\section{Bon Secour Bays, Alabama}

By Marci E. Marot and Christopher G. Smith

\section{Introduction}

The focus of this study was to determine the extent of natural and (or) anthropogenic impacts on the sedimentary records of Mobile and Bon Secour Bays, Alabama during the last 150 years (fig. 1). These bays are unique in that anthropogenic activities are generally widespread and span both the eastern and western shorelines. However, there is a clear distinction in the types of human development and infrastructure between the western and eastern shorelines. These activities and the differences in land-use and -change influence the overall supply and remobilization of sediment to and within the bay. These factors could subsequently threaten the health and integrity of these environments and their ability to mitigate against long-term processes associated with climate change. In an attempt to characterize long-term accretion rates within the Mobile Bay Estuarine System (MBES), seven box cores were collected (fig. 1) and analyzed for excess lead-210 $\left({ }^{210} \mathrm{~Pb}_{\mathrm{xs}}\right.$, the difference between total and supported $\left.{ }^{210} \mathrm{~Pb}\right)$ and cesium-137 $\left({ }^{137} \mathrm{Cs}\right)$ activities. The MBES receives sediment and water from the Alabama and Tombigbee River watersheds, which converge into the Mobile-Tensaw River (MTR) system just prior to discharging into Mobile Bay. Riverine discharge from the MTR system to the bay is second only to the Mississippi River discharge to the Gulf of Mexico for the conterminous United States. 


\section{Data Collection and Processing}

Collection and processing of box cores

During an August 2010 research cruise aboard the U.S. Geological Survey (USGS) RV G.K. Gilbert, box cores were also collected from several stations (table 1; fig. 1) using a modified Wildcotype box corer. After placing the box corer on deck, a 6-inch plastic core liner was inserted into the box core, and the subcore was carefully extracted to preserve the sediment-water interface. The sediment cores were refrigerated after collection to minimize dissolution of the foraminifera tests (see K.A. Richwine and L.E. Osterman, in press) and were subsequently extruded and sampled at 1-centimeter $(\mathrm{cm})$ intervals within 48 hours (h) after collection. The sediment samples were refrigerated and transported to the USGS in St. Petersburg, Florida. The samples remained refrigerated until lab processing.

A split of each 1-cm subsample was processed for basic sediment characteristics (dry bulk density and mass-water content $)$ and geochronology via excess lead-210 $\left({ }^{210} \mathrm{~Pb}_{\mathrm{xs}},\right)$ and ${ }^{137} \mathrm{Cs}$. Masswater content and bulk density were determined by drying 30 milliliters $(\mathrm{mL})$ of wet sediment at 60 degrees Celcius $\left({ }^{\circ} \mathrm{C}\right)$. Differences in the pre- and post-drying masses were used to compute water content; dry bulk density was computed as the ratio of dry sediment to initial volume $(30 \mathrm{~mL})$. Organic matter content was determined using standard loss-on-ignition (LOI); 5 to 10 grams (g) of dried sediment were baked at $450^{\circ} \mathrm{C}$ for $6 \mathrm{~h}$. Pre- and post-baking masses were used to compute a percent LOI.

\section{Gamma Spectroscopy Analyses}

An additional split of each subsample was processed for the determination of ${ }^{210} \mathrm{~Pb}_{\mathrm{xs}}$ and ${ }^{137} \mathrm{Cs}$ by standard gamma-ray spectrometry. Each subsample (approximately $80 \mathrm{~g}$ of wet sediment) was 
freeze dried and subsequently homogenized using a mortar and pestle. Between 15 and $20 \mathrm{~g}$ of dried sediment was sealed in air-tight plastic containers for approximately 4 weeks prior to measurement to achieve secular equilibrium between radon-222 $\left({ }^{222} \mathrm{Rn}\right)$ and its parent radium-226 $\left({ }^{226} \mathrm{Ra}\right)$. Each sample was counted for a 2- to 3-day period on a planar-style, low-energy, high-purity germanium, gamma-ray spectrometer (Canberra, Inc.). Total ${ }^{210} \mathrm{~Pb}$ and ${ }^{137} \mathrm{Cs}$ were determined using photopeaks 46.5 and 661.6 kiloelectron-volts (keV), respectively. Supported ${ }^{210} \mathrm{~Pb}$ (produced from sediment-bound ${ }^{226} \mathrm{Ra}$ ) was estimated using the 295.1 and $352.1 \mathrm{keV}$ photopeaks for ${ }^{214} \mathrm{~Pb}$ and the $609.3 \mathrm{keV}$ photopeak for bismuth-214 $\left({ }^{214} \mathrm{Bi}\right)$. Sample count rates were corrected for detector efficiency (referenced to the International Atomic Energy Agency RGU-1 standard), standard photopeak-intensity, and selfabsorption corrections using a ${ }^{238} \mathrm{U}$ sealed source (Cutshall and others, 1983). The reported activities were decay corrected to the date of sample collection. The statistical counting error for each radioisotope is reported. The critical level $\left(\mathrm{L}_{c}\right)$ (McCurdy and others, 2008) is reported for each radioisotope. The $\mathrm{L}_{\mathrm{c}}$ is the threshold value above which the radioisotope is considered to be detected. Radioisotopic activities were also determined for thorium-234 $\left({ }^{234} \mathrm{Th}, 63.3 \mathrm{keV}\right.$ photopeak) and potassium-40 $\left({ }^{40} \mathrm{~K}, 1460.8 \mathrm{keV}\right.$ photopeak $)$.

\section{Alpha Spectroscopy Analyses}

A split was taken of each subsample for the determination of ${ }^{210} \mathrm{~Pb}_{\text {total }}$ by standard alpha spectrometry (Robbins and Edgington, 1975; Swarzenski and others, 2006). Total ${ }^{210} \mathrm{~Pb}\left({ }^{210} \mathrm{~Pb}_{\text {tot }}=\right.$ $\left.{ }^{210} \mathrm{~Pb}_{\text {sup }}+{ }^{210} \mathrm{~Pb}_{\mathrm{xs}}\right)$ was determined by alpha spectroscopy following the procedure of Robbins and Edgington (1975) and Swarzenski and others (2006). Total ${ }^{210} \mathrm{~Pb}$ activity was determined by measuring its daughter isotope, polonium-210 $\left({ }^{210} \mathrm{Po}\right)$, which is assumed to be in secular equilibrium with its parent. Approximately $5 \mathrm{~g}$ of sediment was spiked with $1 \mathrm{~mL}$ of ${ }^{209}$ Po of known activity (2.70 picocuries per milliliter). Sediments were leached with a combination of concentrated nitric and 
hydrochloric acid with the addition of 30 percent hydrogen peroxide to remove organics. Sediment was taken to near dryness and re-saturated three times with hydrochloric acid until all nitric acid was removed. ${ }^{209,210}$ Po were electroplated onto silver planchets and counted on an Ortec 576A alpha spectrometer. Count rate efficiency for ${ }^{209}$ Po was determined and applied to ${ }^{210} \mathrm{Po}$ counts.

\section{Results and Discussion}

Basic physical (wet and dry bulk density, water content, and loss-on-ignition) and radioanalytical data for the seven box-core sediments are presented in tables $2-8$. The physical characteristics of the sediment show a generally fine-upward pattern for much of the bay, with water content and organic matter content increasing upcore and bulk density decreasing upcore (tables $2-8$ ). Exception to the generalization is observed at MB0810-2BC and -5BC, which lie at the outer edge of the bayhead delta (foredelta). Excess ${ }^{210} \mathrm{~Pb}$ varied considerably across the entire bay. Surface activities vary from 1.18 to $3.24 \mathrm{pCi} \mathrm{g}^{-1}$ and increase away from the bayhead delta. Excess ${ }^{210} \mathrm{~Pb}$ observed at depth in the sediments also varies between $21 \mathrm{~cm}$ (MB0810-5BC) and $45 \mathrm{~cm}$ (MB0810-2BC). With the exception of the most marine-influenced site (MB0810-8BC), average surface ${ }^{210} \mathrm{~Pb}$ activities $\left(2.09 \pm 0.59 \mathrm{pCi} \mathrm{g}^{-1}\right)$ are within error to those observed in local marshes $\left(1.57 \pm 0.50 \mathrm{pCi} \mathrm{g}^{-1}\right.$; C.G. Smith, in press, 2012). In contrast, MB0810-8BC at the bay-gulf inlet has twice the surface activity, suggesting potential focusing from the marine and(or) estuarine system. All of the cores contain quantifiable ${ }^{137} \mathrm{Cs}$; however, the overall preservation of the typical step (dirac delta) function representative of the mid- $20^{\text {th }}$-century test period is not well defined. Instead, ${ }^{137} \mathrm{Cs}$ is distributed uniformly to variable depths $(8-33 \mathrm{~cm})$ within the cores, indicating possible post-depositional mixing. For most cores, the vertical distribution of ${ }^{234} \mathrm{Th}$ and ${ }^{40} \mathrm{~K}$ is fairly uniform (relative standard deviation (RSD) less than 10 percent of the mean). The exceptions are MB0810-8BC and $-20 \mathrm{BC} ;{ }^{234} \mathrm{Th}$ increases at depth in each core. The range in vertically averaged ${ }^{234} \mathrm{Th}$ activities is 1.03 to $1.60 \mathrm{pCi} \mathrm{g}^{-1}$ and 
decreases along the axis of the bay. This trend holds whether MB08010-8BC and -20BC are included or excluded. In comparison, all cores that have RSD of vertically averaged ${ }^{40} \mathrm{~K}$ are all less than 10 percent. Vertically averaged ${ }^{40} \mathrm{~K}$ values are also gradational, with activities decreasing from $12.99 \mathrm{pCi}$ $\mathrm{g}^{-1}$ at the head of the bay (MB0810-2BC) to $10.45 \mathrm{pCi} \mathrm{g}^{-1}$ (average of MB0810-7BC and -8BC) at the foot of the bay. This spatial variability in both ${ }^{234} \mathrm{Th}$ and ${ }^{40} \mathrm{~K}$ may reflect a change in sediment source or composition, the prevailing salinity gradient within the bay, and (or) diagenetic variability within the sediment column.

\section{Acknowledgments}

The authors wish to thank Caitlin Reynolds (USGS) and Andrew Atchinson (Eckerd College) for help with field sampling, and Tim Dellapenna (Texas A\&M Univeristy-Galveston) for graciously allowing the use of the box corer. Assistance in sample processing and data management was provided by Adis Muslic, Tom Harmon, Joseph “Donny” Smoak (University of South Florida-St. Petersburg), and Lisa Osterman (USGS).

\section{References Cited}

Cutshall, N.H., Larsen, I.L., Olsen, C.R., 1983, Direct analysis of ${ }^{210} \mathrm{~Pb}$ in sediment samples: Self absorption corrections: Nuclear Instruments and Methods, v.206, p.309-312.

McCurdy, D.E., Garbarino, J.R., and Mullin, A.H., 2008, Interpreting and reporting radiological water-quality data: U.S. Geological Survey Techniques and Methods, book 5, chap. B6, 33 p.

Richwine, K.A., and Osterman, L.E., 2012, Benthic foraminiferal census data from Mobile Bay, AL Counts of surface samples and box cores: U.S. Geological Survey Data Series 704, available only online at http://pubs.usgs.gov/ds/704/.

Robbins, J.A., and Edgington, D.N., 1975, Determination of recent sedimentation rates in Lake 
Michigan using Pb-210 and Cs-137: Geochimica et Cosmochimica Acta, v.39 no.3, p.285-304.

Smith, C.G., Osterman, L.E., Poore, R.Z., in press, An examination of historic inorganic sedimentation and organic matter accumulation in several marsh types within the Mobile Bay and Mobile-Tensaw River Delta region. Journal of Coastal Research.

Swarzenski, P., Baskaran, M., Rosenbauer, R. and Orem, W., 2006, Historical trace element distribution in sediments from the Mississippi River Delta: Estuaries and Coasts, v.29 no.6, p.1094-1107. 


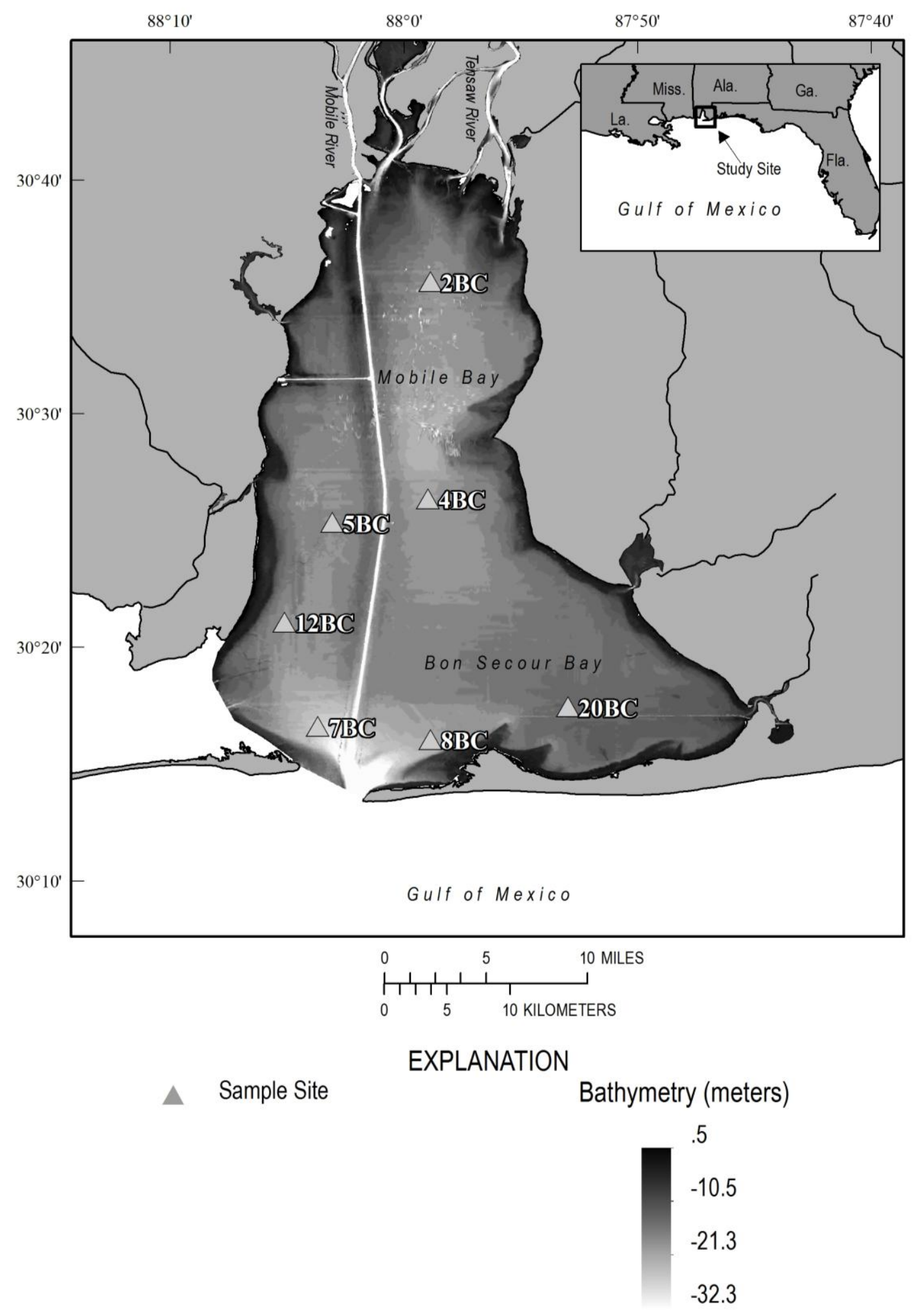

Figure 1. Site locations in Mobile and Bon Secour Bays, Alabama. 
Table 1. Site Location, Mobile and Bon Secour Bays, Alabama.

[dd, decimal degrees; CDT, Cental Daylight Time; m, meter; ${ }^{\circ} \mathrm{C}$, degrees Celsius; salinity, practical salinity unit; --, not measured]

\begin{tabular}{ccccccc}
\hline Sample ID & $\begin{array}{c}\text { Latitude } \\
\text { (dd) }\end{array}$ & $\begin{array}{c}\text { Longitude } \\
\text { (dd) }\end{array}$ & $\begin{array}{c}\text { Date/Time } \\
(\mathbf{C D T})\end{array}$ & $\begin{array}{c}\text { Water } \\
\text { Depth } \\
(\mathbf{m})\end{array}$ & $\begin{array}{c}\text { Temperature } \\
\left({ }^{\circ} \mathbf{C}\right)\end{array}$ & Salinity \\
\hline MB0810-2BC & 30.59440 & 87.98105 & $8 / 6 / 1010: 45$ & 3.7 & 30.66 & 8.09 \\
MB0810-4BC & 30.43943 & 87.98302 & $8 / 6 / 1015: 25$ & 3.8 & 30.35 & 16.43 \\
MB0810-5BC & 30.42261 & 88.05108 & $8 / 6 / 1016: 08$ & 3.8 & 30.48 & 16.49 \\
MB0810-7BC & 30.27719 & 88.06123 & $8 / 5 / 1014: 00$ & 4.9 & 28.73 & 21.36 \\
MB0810-8BC & 30.26765 & 87.98110 & $8 / 5 / 1015: 30$ & 4.1 & 29.30 & 22.74 \\
MB0810-12BC & 30.35144 & 88.08526 & $8 / 5 / 1013: 30$ & 3.8 & 30.80 & 17.52 \\
MB0810-20BC & 30.29100 & 87.88300 & $8 / 5 / 108: 45$ & -- & 31.03 & 16.45 \\
\hline
\end{tabular}


Table 2. Physical and radioisotopic data for Mobile Bay core MB0810-2BC.

[cm, centimeter; $\mathrm{g} / \mathrm{cm}^{3}$, grams per cubic centimeter; \% Mass, percent mass; g OM/g sed, grams of organic matter per grams of sediment; $\mathrm{pCi} / \mathrm{g}$, picocuries per gram; ND, not detected; $\mathrm{L}_{\mathrm{c}}$, critical level]

\begin{tabular}{|c|c|c|c|c|c|c|c|c|c|c|}
\hline $\begin{array}{l}\text { Depth } \\
(\mathrm{cm})\end{array}$ & $\begin{array}{c}\text { Wet Bulk } \\
\text { Density } \\
\left(\mathrm{g} / \mathrm{cm}^{3}\right)\end{array}$ & $\begin{array}{c}\text { Dry Bulk } \\
\text { Density } \\
\left(\mathrm{g} / \mathrm{cm}^{3}\right)\end{array}$ & $\begin{array}{c}\text { Water } \\
\text { Content } \\
\text { (\% Mass) }\end{array}$ & $\begin{array}{l}\text { Loss On } \\
\text { Ignition } \\
\text { OM/g sed) }\end{array}$ & $\mathrm{Pb}-210^{\mathrm{a}} \quad(\mathrm{pCi} / \mathrm{g})$ & $\begin{array}{l}\text { Cs-137 } \\
(\mathrm{pCi} / \mathrm{g})\end{array}$ & $\begin{array}{l}\mathrm{Pb}-210^{\mathrm{b}} \\
(\mathrm{pCCi} / \mathrm{g})\end{array}$ & $\begin{array}{r}\mathrm{Ra}-226^{\mathrm{b}} \\
(\mathrm{pCi} / \mathrm{g})\end{array}$ & Th-234 ${ }^{b} \quad(p C i / g)$ & $\begin{array}{l}\mathrm{K}-40^{\mathrm{b}} \\
(\mathrm{pCi} / \mathrm{g})\end{array}$ \\
\hline $0-1$ & 1.23 & 0.55 & 0.55 & 9.01 & $1.18+/-0.09$ & ND & $1.99+/-0.17$ & $0.94+/-0.06$ & $1.65+/-0.19$ & $12.46+/-0.91$ \\
\hline $1-2$ & 1.23 & 0.57 & 0.53 & 7.81 & $1.39+/-0.01$ & ND & $2.20+/-0.17$ & $1.04+/-0.05$ & $1.39+/-0.16$ & $12.53+/-0.85$ \\
\hline $2-3$ & 1.21 & 0.55 & 0.55 & 8.35 & $1.58+/-0.02$ & $0.10+/-0.02$ & $2.22+/-0.13$ & $0.99+/-0.04$ & $1.43+/-0.13$ & $12.44+/-0.65$ \\
\hline $3-4$ & 1.19 & 0.50 & 0.58 & 8.93 & $1.45+/-0.02$ & $0.08+/-0.03$ & $2.19+/-0.17$ & $0.99+/-0.05$ & $1.53+/-0.19$ & $12.07+/-0.83$ \\
\hline $4-5$ & 1.20 & 0.56 & 0.54 & 9.08 & $1.57+/-0.14$ & $0.10+/-0.03$ & $2.14+/-0.14$ & $0.98+/-0.05$ & $1.51+/-0.15$ & $13.42+/-0.76$ \\
\hline $\begin{array}{l}5-6 \\
5-6^{c}\end{array}$ & 1.14 & 0.47 & 0.59 & $\begin{array}{l}8.62 \\
8.67\end{array}$ & $\begin{array}{l}1.39+/-0.06 \\
1.50+/-0.02\end{array}$ & $0.08+/-0.03$ & $2.28+/-0.17$ & $0.96+/-0.05$ & $1.42+/-0.18$ & $12.95+/-0.83$ \\
\hline $6-7$ & 1.17 & 0.47 & 0.59 & 8.84 & $1.45+/-0.11$ & $0.11+/-0.03$ & $2.35+/-0.18$ & $1.01+/-0.06$ & $1.52+/-0.19$ & $12.57+/-0.86$ \\
\hline $7-8$ & 1.19 & 0.49 & 0.59 & 8.36 & $1.47+/-0.04$ & $0.09+/-0.02$ & $2.01+/-0.15$ & $0.88+/-0.05$ & $1.30+/-0.16$ & $11.96+/-0.73$ \\
\hline $8-9$ & 1.19 & 0.51 & 0.58 & 7.13 & $1.48+/-0.02$ & $0.10+/-0.02$ & $2.25+/-0.15$ & $0.91+/-0.04$ & $1.42+/-0.15$ & $12.95+/-0.73$ \\
\hline $9-10$ & 1.16 & 0.49 & 0.58 & 8.39 & $1.40+/-0.02$ & $0.09+/-0.02$ & $1.94+/-0.12$ & $0.92+/-0.04$ & $1.54+/-0.13$ & $11.71+/-0.61$ \\
\hline $10-11$ & 1.19 & 0.53 & 0.55 & 8.85 & $1.57+/-0.10$ & $0.10+/-0.03$ & $2.17+/-0.17$ & $0.90+/-0.05$ & $1.40+/-0.17$ & $12.59+/-0.85$ \\
\hline $11-12$ & 1.15 & 0.48 & 0.58 & 8.75 & $1.51+/-0.13$ & $0.11+/-0.03$ & $2.20+/-0.17$ & $0.91+/-0.05$ & $1.32+/-0.18$ & $12.80+/-0.89$ \\
\hline $11-12^{\mathrm{c}}$ & & & & 8.49 & $1.43+/-0.04$ & & & & & \\
\hline $12-13$ & 1.15 & 0.47 & 0.59 & 8.51 & $1.57+/-0.14$ & $0.10+/-0.02$ & $2.41+/-0.13$ & $0.90+/-0.04$ & $1.55+/-0.14$ & $12.74+/-0.63$ \\
\hline $13-14$ & 1.22 & 0.55 & 0.55 & 7.90 & $1.55+/-0.21$ & $0.07+/-0.02$ & $1.92+/-0.14$ & $0.78+/-0.04$ & $1.30+/-0.15$ & $10.62+/-0.68$ \\
\hline $14-15$ & 1.16 & 0.49 & 0.58 & 8.31 & $1.55+/-0.01$ & $0.09+/-0.02$ & $2.06+/-0.14$ & $0.94+/-0.04$ & $1.39+/-0.15$ & $11.75+/-0.69$ \\
\hline $15-16$ & 1.17 & 0.48 & 0.59 & 8.47 & $1.57+/-0.20$ & $0.11+/-0.03$ & $2.19+/-0.15$ & $0.94+/-0.05$ & $1.41+/-0.15$ & $12.65+/-0.73$ \\
\hline $16-17$ & 1.18 & 0.51 & 0.56 & 7.86 & $1.41+/-0.10$ & $0.08+/-0.02$ & $2.01+/-0.13$ & $0.86+/-0.04$ & $1.61+/-0.13$ & $12.20+/-0.62$ \\
\hline $17-18$ & 1.27 & 0.64 & 0.49 & 7.54 & $1.25+/-0.10$ & $0.08+/-0.03$ & $2.03+/-0.17$ & $1.01+/-0.05$ & $1.63+/-0.19$ & $12.11+/-0.85$ \\
\hline $17-18^{\mathrm{c}}$ & & & & 7.04 & $1.24+/-0.12$ & & & & & \\
\hline $18-19$ & 1.18 & 0.54 & 0.54 & 6.50 & $1.19+/-0.20$ & $0.09+/-0.03$ & $1.91+/-0.14$ & $0.97+/-0.05$ & $1.54+/-0.15$ & $12.52+/-0.74$ \\
\hline $19-20$ & 1.23 & 0.61 & 0.51 & 5.84 & $0.99+/-0.13$ & $0.07+/-0.03$ & $1.90+/-0.15$ & $0.97+/-0.05$ & $1.69+/-0.20$ & $11.69+/-0.82$ \\
\hline $20-21$ & 1.30 & 0.67 & 0.48 & 5.79 & $1.03+/-0.04$ & $0.10+/-0.03$ & $1.87+/-0.17$ & $1.09+/-0.06$ & $1.47+/-0.19$ & $13.93+/-0.90$ \\
\hline $21-22$ & 1.27 & 0.67 & 0.47 & 7.03 & $0.90+/-0.06$ & $0.06+/-0.03$ & $1.86+/-0.15$ & $1.09+/-0.05$ & $1.74+/-0.18$ & $12.71+/-0.76$ \\
\hline $22-23$ & 1.30 & 0.70 & 0.47 & 6.49 & $0.87+/-0.05$ & $0.12+/-0.03$ & $1.58+/-0.16$ & $1.09+/-0.06$ & $1.55+/-0.20$ & $11.91+/-0.85$ \\
\hline $23-24$ & 1.27 & 0.61 & 0.52 & 6.91 & $0.81+/-0.05$ & $0.11+/-0.02$ & $1.54+/-0.10$ & $1.06+/-0.04$ & $1.85+/-0.14$ & $12.97+/-0.58$ \\
\hline $23-24^{\mathrm{c}}$ & & & & 7.06 & $0.81+/-0.04$ & & & & & \\
\hline $24-25$ & 1.24 & 0.58 & 0.53 & 7.70 & $0.84+/-0.01$ & $0.13+/-0.03$ & $1.62+/-0.16$ & $1.00+/-0.05$ & $1.82+/-0.19$ & $13.66+/-0.89$ \\
\hline $25-26$ & 1.24 & 0.57 & 3.93 & 7.60 & $0.84+/-0.10$ & $0.10+/-0.03$ & $1.43+/-0.16$ & $0.95+/-0.05$ & $1.71+/-0.20$ & $13.01+/-0.89$ \\
\hline $26-27$ & 1.23 & 0.58 & 0.53 & 7.41 & $0.77+/-0.06$ & $0.07+/-0.03$ & $1.43+/-0.13$ & $1.04+/-0.05$ & $1.68+/-0.17$ & $13.25+/-0.77$ \\
\hline $27-28$ & 1.21 & 0.55 & 0.54 & 8.02 & $0.77+/-0.07$ & $0.10+/-0.03$ & $1.44+/-0.16$ & $0.95+/-0.05$ & $1.93+/-0.20$ & $13.14+/-0.92$ \\
\hline $28-29$ & 1.16 & 0.50 & 0.56 & 8.46 & $0.76+/-0.01$ & $0.07+/-0.03$ & $1.39+/-0.14$ & $0.94+/-0.05$ & $1.99+/-0.17$ & $14.81+/-0.79$ \\
\hline $29-30$ & 1.19 & 0.54 & 0.55 & 8.44 & $0.68+/-0.01$ & $0.05+/-0.03$ & $1.31+/-0.16$ & $0.89+/-0.05$ & $1.81+/-0.19$ & $14.84+/-0.91$ \\
\hline $30-31$ & 1.16 & 0.48 & 0.58 & 8.25 & $0.62+/-0.01$ & ND & $1.09+/-0.15$ & $0.81+/-0.05$ & $1.86+/-0.19$ & $13.75+/-0.92$ \\
\hline $31-32$ & 1.16 & 0.49 & 0.58 & 8.45 & $0.68+/-0.01$ & ND & $1.24+/-0.17$ & $0.90+/-0.06$ & $2.00+/-0.21$ & $14.59+/-0.97$ \\
\hline $32-33$ & 1.20 & 0.54 & 0.55 & 8.53 & $0.61+/-0.10$ & ND & $0.93+/-0.13$ & $0.87+/-0.05$ & $1.69+/-0.17$ & $14.33+/-0.80$ \\
\hline $33-34$ & 1.15 & 0.48 & 0.58 & 8.45 & $0.70+/-0.01$ & ND & $1.12+/-0.16$ & $0.85+/-0.05$ & $1.93+/-0.20$ & $13.61+/-0.92$ \\
\hline $34-35$ & 1.18 & 0.50 & 0.58 & 8.54 & $0.84+/-0.10$ & $0.09+/-0.03$ & $1.36+/-0.15$ & $0.92+/-0.05$ & $1.80+/-0.20$ & $14.20+/-0.92$ \\
\hline $35-36$ & 1.17 & 0.50 & 0.57 & 8.89 & $0.91+/-0.02$ & $0.13+/-0.03$ & $1.37+/-0.13$ & $0.87+/-0.04$ & $1.65+/-0.17$ & $13.11+/-0.75$ \\
\hline $36-37$ & 1.19 & 0.54 & 0.55 & 8.95 & $0.96+/-0.04$ & $0.14+/-0.03$ & $1.56+/-0.16$ & $0.88+/-0.05$ & $1.48+/-0.19$ & $14.03+/-0.92$ \\
\hline $36-37^{\mathrm{c}}$ & & & & 8.75 & $0.87+/-0.04$ & & & & & \\
\hline $37-38$ & 1.19 & 0.54 & 0.55 & 8.89 & $0.99+/-0.05$ & $0.11+/-0.03$ & $1.38+/-0.17$ & $0.89+/-0.05$ & $1.40+/-0.19$ & $12.62+/-0.89$ \\
\hline $38-39$ & 1.22 & 0.57 & 0.53 & 8.68 & $1.08+/-0.04$ & $0.14+/-0.03$ & $1.45+/-0.12$ & $0.89+/-0.04$ & $1.46+/-0.15$ & $13.92+/-0.76$ \\
\hline $39-40$ & 1.21 & 0.59 & 0.51 & 6.97 & $0.87+/-0.02$ & $0.11+/-0.03$ & $1.31+/-0.13$ & $0.96+/-0.04$ & $1.46+/-0.16$ & $13.21+/-0.73$ \\
\hline $40-41$ & 1.18 & 0.56 & 0.53 & 6.80 & $0.79+/-0.08$ & $0.13+/-0.03$ & $1.34+/-0.13$ & $0.97+/-0.04$ & $1.71+/-0.16$ & $13.65+/-0.73$ \\
\hline $41-42$ & 1.15 & 0.51 & 0.56 & 6.89 & $0.68+/-0.03$ & $0.09+/-0.02$ & $1.27+/-0.11$ & $0.92+/-0.04$ & $1.79+/-0.14$ & $13.47+/-0.64$ \\
\hline $42-43$ & 1.25 & 0.61 & 0.51 & 6.84 & $0.70+/-0.18$ & $0.06+/-0.02$ & $1.26+/-0.13$ & $0.91+/-0.04$ & $1.70+/-0.17$ & $13.06+/-0.75$ \\
\hline $43-44$ & 1.26 & 0.62 & 0.50 & 6.55 & $0.71+/-0.01$ & $0.12+/-0.03$ & $1.41+/-0.14$ & $0.94+/-0.05$ & $1.62+/-0.17$ & $13.55+/-0.78$ \\
\hline $43-44^{\mathrm{c}}$ & & & & 6.47 & $0.75+/-0.10$ & & & & & \\
\hline $44-45$ & 1.26 & 0.64 & 0.49 & 5.79 & $0.75+/-0.02$ & $0.15+/-0.02$ & $1.47+/-0.11$ & $1.02+/-0.04$ & $1.69+/-0.15$ & $12.88+/-0.63$ \\
\hline
\end{tabular}

$\mathrm{a}=$ results determined by alpha spectroscopy method; $\mathrm{b}=$ results determined by gamma spectroscopy method; $\mathrm{c}=$ replicate analysis

$\mathrm{L}_{\mathrm{c}: \mathrm{Cs}-137}=0.02 \mathrm{pCi} / \mathrm{g} ; \mathrm{L}_{\mathrm{c}: \mathrm{Pb}-210}=0.09 \mathrm{pCi} / \mathrm{g} ; \mathrm{L}_{\mathrm{c}: \mathrm{Ra}-226}=0.05 \mathrm{pCi} / \mathrm{g} ; \mathrm{L}_{\mathrm{c}: T h-234}=0.12 \mathrm{pCi} / \mathrm{g} ; \mathrm{L}_{\mathrm{c}: \mathrm{K}-40}=0.31 \mathrm{pCi} / \mathrm{g}$ 
Table 3. Physical and radioisotopic data for Mobile Bay core MB0810-4BC.

[cm, centimeter; $\mathrm{g} / \mathrm{cm}^{3}$, grams per cubic centimeter; \% Mass, percent mass; g OM/g sed, grams of organic matter per grams of sediment; pCi/g, picocuries per gram; ND, not detected; $L_{c}$, critical level]

\begin{tabular}{|c|c|c|c|c|c|c|c|c|c|c|}
\hline Depth $\quad(\mathrm{cm})$ & $\begin{array}{c}\text { Wet Bulk } \\
\text { Density } \\
\left(\mathrm{g} / \mathrm{cm}^{3}\right)\end{array}$ & $\begin{array}{c}\text { Dry Bulk } \\
\text { Density } \\
\left(\mathrm{g} / \mathrm{cm}^{3}\right)\end{array}$ & $\begin{array}{c}\text { Water } \\
\text { Content } \\
\text { (\% Mass) }\end{array}$ & $\begin{array}{l}\text { Loss On } \\
\text { Ignition } \\
\text { OM/g sed) }\end{array}$ & $\mathrm{Pb}-210^{\mathrm{a}} \quad(\mathrm{pCi} / \mathrm{g})$ & $\begin{array}{l}\mathrm{Cs}-137^{\mathrm{b}} \\
(\mathrm{pCi} / \mathrm{g})\end{array}$ & $\begin{array}{l}\mathrm{Pb}-210^{\mathrm{b}} \\
(\mathrm{pCi} / \mathrm{g})\end{array}$ & $\begin{array}{l}\mathrm{Ra}-226^{\mathrm{b}} \\
(\mathrm{pCi} / \mathrm{g})\end{array}$ & Th-234 $4^{\mathrm{b}} \quad(\mathrm{pCi} / \mathrm{g})$ & $\begin{array}{l}\mathrm{K}-40^{\mathrm{b}} \\
(\mathrm{pCi} / \mathrm{g})\end{array}$ \\
\hline $0-1$ & 1.20 & 0.36 & 0.70 & 13.52 & $1.76+/-0.03$ & $0.10+/-0.03$ & $2.49+/-0.15$ & $0.78+/-0.04$ & $1.22+/-0.13$ & $14.79+/-0.84$ \\
\hline $1-2$ & 1.15 & 0.33 & 0.72 & 13.84 & $1.47+/-0.03$ & $0.10+/-0.02$ & $2.74+/-0.12$ & $0.79+/-0.03$ & $1.40+/-0.12$ & $12.68+/-0.55$ \\
\hline $2-3$ & 1.22 & 0.41 & 0.67 & 12.66 & $1.79+/-0.03$ & $0.11+/-0.02$ & $2.78+/-0.11$ & $0.77+/-0.03$ & $1.40+/-0.10$ & $12.47+/-0.50$ \\
\hline $3-4$ & 1.24 & 0.40 & 0.68 & 13.87 & $1.68+/-0.03$ & $0.10+/-0.02$ & $2.59+/-0.11$ & $0.76+/-0.03$ & $1.48+/-0.11$ & $12.63+/-0.55$ \\
\hline $4-5$ & 1.22 & 0.41 & 0.66 & 14.99 & $1.77+/-0.03$ & $0.09+/-0.02$ & $2.73+/-0.14$ & $0.79+/-0.04$ & $1.43+/-0.13$ & $12.77+/-0.62$ \\
\hline $5-6$ & 1.19 & 0.39 & 0.67 & 13.19 & $1.34+/-0.03$ & $0.12+/-0.02$ & $2.79+/-0.12$ & $0.80+/-0.04$ & $1.46+/-0.13$ & $15.28+/-0.71$ \\
\hline $6-7$ & 1.20 & 0.37 & 0.69 & 13.35 & $1.53+/-0.03$ & $0.10+/-0.02$ & $2.71+/-0.12$ & $0.78+/-0.04$ & $1.44+/-0.12$ & $13.13+/-0.61$ \\
\hline $7-8$ & 1.23 & 0.43 & 0.65 & 12.92 & $1.48+/-0.03$ & $0.10+/-0.02$ & $2.71+/-0.13$ & $0.77+/-0.04$ & $1.32+/-0.12$ & $12.66+/-0.63$ \\
\hline $8-9$ & 1.22 & 0.42 & 0.66 & 12.54 & $1.68+/-0.03$ & $0.11+/-0.02$ & $2.80+/-0.11$ & $0.77+/-0.03$ & $1.49+/-0.11$ & $12.67+/-0.51$ \\
\hline $9-10$ & 1.26 & 0.48 & 0.62 & 12.61 & $1.79+/-0.02$ & $0.12+/-0.02$ & $2.68+/-0.14$ & $0.74+/-0.04$ & $1.47+/-0.14$ & $12.80+/-0.69$ \\
\hline $10-11$ & 1.21 & 0.42 & 0.65 & 12.07 & $1.67+/-0.03$ & $0.10+/-0.02$ & $2.67+/-0.13$ & $0.82+/-0.04$ & $1.55+/-0.13$ & $11.74+/-0.62$ \\
\hline $11-12$ & 1.21 & 0.47 & 0.62 & 11.72 & $1.57+/-0.03$ & $0.11+/-0.02$ & $2.51+/-0.13$ & $0.84+/-0.04$ & $1.46+/-0.14$ & $12.69+/-0.69$ \\
\hline $11-12^{\mathrm{c}}$ & & & & 11.63 & $1.44+/-0.03$ & & & & & \\
\hline $12-13$ & 1.22 & 0.45 & 0.63 & 13.04 & $1.21+/-0.02$ & $0.11+/-0.02$ & $2.28+/-0.11$ & $0.79+/-0.03$ & $1.73+/-0.13$ & $11.72+/-0.56$ \\
\hline $13-14$ & 1.28 & 0.51 & 0.60 & 11.82 & $1.19+/-0.02$ & $0.09+/-0.03$ & $2.13+/-0.14$ & $0.82+/-0.04$ & $1.66+/-0.14$ & $11.91+/-0.68$ \\
\hline $14-15$ & 1.31 & 0.53 & 0.59 & 11.57 & $1.09+/-0.02$ & $0.08+/-0.02$ & $1.79+/-0.11$ & $0.86+/-0.04$ & $1.67+/-0.12$ & $12.79+/-0.57$ \\
\hline $15-16$ & 1.30 & 0.56 & 0.57 & 11.54 & $1.04+/-0.02$ & $0.05+/-0.03$ & $1.71+/-0.15$ & $0.85+/-0.05$ & $1.78+/-0.18$ & $12.22+/-0.83$ \\
\hline $16-17$ & 1.30 & 0.56 & 0.57 & 11.80 & $0.60+/-0.03$ & $0.08+/-0.02$ & $1.47+/-0.13$ & $0.84+/-0.04$ & $1.72+/-0.14$ & $12.95+/-0.74$ \\
\hline $17-18$ & 1.30 & 0.58 & 0.55 & 11.77 & $0.93+/-0.03$ & $0.07+/-0.02$ & $1.34+/-0.13$ & $0.82+/-0.04$ & $1.68+/-0.15$ & $12.49+/-0.71$ \\
\hline $17-18^{\mathrm{c}}$ & & & & 11.55 & $1.00+/-0.03$ & & & & & \\
\hline $18-19$ & 1.33 & 0.58 & 0.56 & 11.75 & $0.70+/-0.02$ & $0.03+/-0.02$ & $1.32+/-0.12$ & $0.85+/-0.04$ & $1.78+/-0.14$ & $12.76+/-0.65$ \\
\hline $19-20$ & 1.31 & 0.60 & 0.54 & 11.94 & $0.73+/-0.03$ & ND & $1.30+/-0.13$ & $0.83+/-0.04$ & $1.74+/-0.15$ & $12.07+/-0.71$ \\
\hline $20-21$ & 1.31 & 0.58 & 0.56 & 11.79 & $0.71+/-0.03$ & ND & $1.31+/-0.21$ & $0.83+/-0.07$ & $1.46+/-0.21$ & $12.29+/-1.10$ \\
\hline $21-22$ & 1.31 & 0.58 & 0.56 & 12.31 & $0.85+/-0.04$ & ND & $1.21+/-0.11$ & $0.79+/-0.04$ & $1.59+/-0.13$ & $12.99+/-0.65$ \\
\hline $22-23$ & 1.30 & 0.56 & 0.57 & 12.18 & $0.57+/-0.03$ & ND & $1.26+/-0.12$ & $0.84+/-0.04$ & $1.68+/-0.14$ & $13.35+/-0.73$ \\
\hline $23-24$ & 1.31 & 0.59 & 0.55 & 12.01 & $0.44+/-0.04$ & ND & $1.14+/-0.13$ & $0.82+/-0.04$ & $1.55+/-0.15$ & $12.74+/-0.74$ \\
\hline $24-25$ & 1.28 & 0.48 & 0.63 & 12.03 & $0.47+/-0.04$ & ND & $1.12+/-0.12$ & $0.76+/-0.04$ & $1.41+/-0.13$ & $12.75+/-0.71$ \\
\hline $24-25^{\mathrm{c}}$ & & & & 11.82 & $0.51+/-0.04$ & & & & & \\
\hline $25-26$ & 1.34 & 0.58 & 0.56 & 11.66 & $0.50+/-0.04$ & ND & $1.04+/-0.09$ & $0.79+/-0.03$ & $1.61+/-0.13$ & $13.12+/-0.59$ \\
\hline $26-27$ & 1.32 & 0.63 & 0.52 & 11.59 & $0.29+/-0.05$ & ND & $1.10+/-0.12$ & $0.84+/-0.04$ & $1.56+/-0.15$ & $13.27+/-0.74$ \\
\hline $27-28$ & 1.29 & 0.53 & 0.59 & 11.32 & $0.54+/-0.03$ & ND & $1.07+/-0.13$ & $0.84+/-0.04$ & $1.56+/-0.16$ & $13.65+/-0.74$ \\
\hline $28-29$ & 1.30 & 0.57 & 0.57 & 11.28 & $0.51+/-0.03$ & ND & $0.97+/-0.14$ & $0.77+/-0.05$ & $1.49+/-0.17$ & $13.72+/-0.88$ \\
\hline $29-30$ & 1.33 & 0.58 & 0.56 & 11.26 & $0.56+/-0.03$ & ND & $0.97+/-0.15$ & $0.83+/-0.05$ & $1.47+/-0.17$ & $12.39+/-0.88$ \\
\hline $30-31$ & 1.33 & 0.58 & 0.57 & 11.38 & $0.55+/-0.03$ & ND & $0.98+/-0.13$ & $0.81+/-0.05$ & $1.56+/-0.16$ & $12.69+/-0.76$ \\
\hline $30-31^{\mathrm{c}}$ & & & & 11.33 & $0.48+/-0.03$ & & & & & \\
\hline $31-32$ & 1.28 & 0.60 & 0.53 & 9.64 & $0.57+/-0.04$ & ND & $0.93+/-0.12$ & $0.77+/-0.04$ & $1.51+/-0.15$ & $13.09+/-0.72$ \\
\hline $32-33$ & 1.32 & 0.59 & 0.56 & 9.64 & $0.52+/-0.03$ & ND & $1.02+/-0.11$ & $0.77+/-0.04$ & $1.58+/-0.13$ & $12.28+/-0.64$ \\
\hline $33-34$ & 1.32 & 0.58 & 0.56 & 9.46 & $0.52+/-0.04$ & ND & $0.90+/-0.14$ & $0.74+/-0.05$ & $1.59+/-0.18$ & $12.63+/-0.80$ \\
\hline $34-35$ & 1.31 & 0.56 & 0.57 & 9.69 & $0.58+/-0.04$ & ND & $1.01+/-0.10$ & $0.76+/-0.03$ & $1.70+/-0.13$ & $14.11+/-0.60$ \\
\hline
\end{tabular}

$\mathrm{a}=$ results determined by alpha spectroscopy method; $\mathrm{b}=$ results determined by gamma spectroscopy method; $\mathrm{c}=$ replicate analysis

$\mathrm{L}_{\mathrm{c}: \mathrm{Cs}-137}=0.02 \mathrm{pCi} / \mathrm{g} ; \mathrm{L}_{\mathrm{c}: \mathrm{Pb}-210}=0.12 \mathrm{pCi} / \mathrm{g} ; \mathrm{L}_{\mathrm{c}: \mathrm{Ra}-226}=0.07 \mathrm{pCi} / \mathrm{g} ; \mathrm{L}_{\mathrm{c}: \mathrm{Th}-234}=0.13 \mathrm{pCi} / \mathrm{g} ; \mathrm{L}_{\mathrm{c}: \mathrm{K}-40}=0.36 \mathrm{pCi} / \mathrm{g}$ 
Table 4. Physical and radioisotopic data for Mobile Bay core MB0810-5BC.

$\left[\mathrm{cm}\right.$, centimeter; $\mathrm{g} / \mathrm{cm}^{3}$, grams per cubic centimeter; \% Mass, percent mass; g OM/g sed, grams of organic matter per grams of sediment; $\mathrm{pCi} / \mathrm{g}$, picocuries per gram; $\mathrm{ND}$, not detected; $\mathrm{L}_{\mathrm{c}}$, critical level]

\begin{tabular}{|c|c|c|c|c|c|c|c|c|c|c|}
\hline Depth & $\begin{array}{c}\text { Wet Bulk } \\
\text { Density } \\
\left(\mathrm{g} / \mathrm{cm}^{3}\right)\end{array}$ & $\begin{array}{c}\text { Dry Bulk } \\
\text { Density } \\
\left(\mathrm{g} / \mathrm{cm}^{3}\right)\end{array}$ & $\begin{array}{c}\text { Water } \\
\text { Content } \\
\text { (\% Mass) }\end{array}$ & $\begin{array}{l}\text { Loss On } \\
\text { Ignition } \\
\text { OM/g sed) }\end{array}$ & $\mathrm{Pb}-210^{\mathrm{a}} \quad(\mathrm{pC} \mathrm{C} / \mathrm{g})$ & $\begin{array}{l}\text { Cs-137 } \\
(\mathrm{pCi} / \mathrm{g})\end{array}$ & $\begin{array}{l}\mathrm{Pb}-210^{\mathrm{b}} \\
(\mathrm{pCi} / \mathrm{g})\end{array}$ & $\begin{array}{r}\mathrm{Ra}-226^{\mathrm{b}} \\
(\mathrm{pCi} / \mathrm{g})\end{array}$ & Th-234 $\quad(p C i / g)$ & $\begin{array}{l}\mathrm{K}-40^{\mathrm{b}} \\
(\mathrm{pCi} / \mathrm{g})\end{array}$ \\
\hline $0-1$ & 1.08 & 0.39 & 0.64 & 10.59 & $1.98+/-0.02$ & $0.06+/-0.02$ & $2.07+/-0.13$ & $0.66+/-0.04$ & $0.91+/-0.13$ & $9.29+/-0.60$ \\
\hline $1-2$ & 1.13 & 0.42 & 0.62 & 10.19 & $1.82+/-0.12$ & $0.09+/-0.02$ & $2.51+/-0.12$ & $0.83+/-0.03$ & $1.40+/-0.12$ & $12.27+/-0.57$ \\
\hline $2-3$ & 1.11 & 0.41 & 0.63 & 9.83 & $1.61+/-0.07$ & $0.08+/-0.03$ & $2.51+/-0.17$ & $0.86+/-0.05$ & $1.45+/-0.16$ & $11.54+/-0.76$ \\
\hline $3-4$ & 1.13 & 0.47 & 0.58 & 9.71 & $1.87+/-0.02$ & $0.07+/-0.03$ & $2.40+/-0.16$ & $0.86+/-0.05$ & $1.40+/-0.16$ & $12.27+/-0.78$ \\
\hline $3-4^{c}$ & & & & 8.15 & $1.92+/-0.03$ & & & & & \\
\hline $4-5$ & 1.12 & 0.43 & 0.62 & 9.86 & $1.85+/-0.15$ & $0.08+/-0.03$ & $2.42+/-0.17$ & $0.84+/-0.05$ & $1.38+/-0.18$ & $11.56+/-0.81$ \\
\hline $5-6$ & 1.16 & 0.45 & 0.61 & 9.82 & $1.68+/-0.04$ & $0.10+/-0.03$ & $2.60+/-0.17$ & $0.88+/-0.05$ & $1.44+/-0.17$ & $11.28+/-0.78$ \\
\hline $6-7$ & 1.10 & 0.40 & 0.63 & 8.75 & $1.34+/-0.03$ & $0.07+/-0.02$ & $2.18+/-0.14$ & $0.78+/-0.04$ & $1.21+/-0.14$ & $10.38+/-0.67$ \\
\hline $7-8$ & 1.15 & 0.46 & 0.60 & 9.61 & $1.10+/-0.08$ & $0.04+/-0.02$ & $1.82+/-0.16$ & $0.89+/-0.05$ & $1.53+/-0.18$ & $12.42+/-0.81$ \\
\hline $8-9$ & 1.18 & 0.51 & 0.57 & 9.14 & $0.87+/-0.05$ & ND & $1.33+/-0.10$ & $0.83+/-0.03$ & $1.52+/-0.12$ & $13.47+/-0.59$ \\
\hline $9-10$ & 1.20 & 0.54 & 0.55 & 9.01 & $0.79+/-0.03$ & ND & $1.15+/-0.13$ & $0.86+/-0.04$ & $1.40+/-0.15$ & $13.50+/-0.72$ \\
\hline $10-11$ & 1.18 & 0.50 & 0.58 & 9.72 & $0.73+/-0.03$ & ND & $1.23+/-0.11$ & $0.89+/-0.04$ & $1.42+/-0.14$ & $14.04+/-0.67$ \\
\hline $11-12$ & 1.16 & 0.49 & 0.58 & 9.99 & $0.89+/-0.02$ & ND & $1.21+/-0.14$ & $0.85+/-0.05$ & $1.39+/-0.17$ & $12.84+/-0.81$ \\
\hline $12-13$ & 1.21 & 0.56 & 0.54 & 9.45 & $0.78+/-0.03$ & ND & $1.08+/-0.15$ & $0.85+/-0.05$ & $1.57+/-0.19$ & $14.07+/-0.91$ \\
\hline $12-13^{\mathrm{c}}$ & & & & 7.83 & $0.63+/-0.05$ & & & & & \\
\hline $13-14$ & 1.18 & 0.52 & 0.55 & 9.29 & $0.67+/-0.15$ & ND & $1.03+/-0.12$ & $0.89+/-0.04$ & $1.46+/-0.16$ & $13.52+/-0.74$ \\
\hline $14-15$ & 1.19 & 0.49 & 0.59 & 10.10 & $0.76+/-0.01$ & ND & $1.10+/-0.12$ & $0.85+/-0.04$ & $1.39+/-0.14$ & $12.82+/-0.73$ \\
\hline $15-16$ & 1.18 & 0.49 & 0.58 & 10.96 & $0.75+/-0.03$ & ND & $1.19+/-0.12$ & $0.86+/-0.04$ & $1.54+/-0.16$ & $13.61+/-0.73$ \\
\hline $16-17$ & 1.17 & 0.45 & 0.61 & 10.62 & $0.73+/-0.01$ & ND & $1.15+/-0.15$ & $0.82+/-0.05$ & $1.36+/-0.18$ & $13.67+/-0.85$ \\
\hline $17-18$ & 1.17 & 0.49 & 0.58 & 9.98 & $0.64+/-0.18$ & ND & $1.03+/-0.16$ & $0.84+/-0.06$ & $1.34+/-0.19$ & $13.39+/-0.95$ \\
\hline $18-19$ & 1.19 & 0.50 & 0.58 & 9.38 & $0.70+/-0.15$ & ND & $1.07+/-0.13$ & $0.82+/-0.04$ & $1.44+/-0.16$ & $12.80+/-0.76$ \\
\hline $19-20$ & 1.21 & 0.50 & 0.59 & 10.10 & $0.78+/-0.08$ & ND & $1.10+/-0.15$ & $0.87+/-0.05$ & $1.31+/-0.19$ & $13.09+/-0.86$ \\
\hline $20-21$ & 1.18 & 0.48 & 0.60 & 10.56 & $0.73+/-0.08$ & ND & $1.14+/-0.17$ & $0.83+/-0.05$ & $1.54+/-0.20$ & $12.61+/-0.89$ \\
\hline $21-22$ & 1.16 & 0.48 & 0.59 & 9.81 & $0.71+/-0.01$ & ND & $1.08+/-0.13$ & $0.85+/-0.04$ & $1.35+/-0.16$ & $13.00+/-0.72$ \\
\hline $22-23$ & 1.18 & 0.48 & 0.60 & 9.45 & $0.78+/-0.04$ & ND & $1.15+/-0.13$ & $0.88+/-0.05$ & $1.41+/-0.17$ & $13.34+/-0.77$ \\
\hline $23-24$ & 1.15 & 0.46 & 0.60 & 9.46 & $0.71+/-0.01$ & ND & $1.17+/-0.11$ & $0.86+/-0.04$ & $1.49+/-0.13$ & $13.08+/-0.63$ \\
\hline $24-25$ & 1.16 & 0.49 & 0.58 & 9.44 & $0.80+/-0.23$ & ND & $1.15+/-0.15$ & $0.87+/-0.05$ & $1.40+/-0.18$ & $12.72+/-0.84$ \\
\hline $24-25^{\mathrm{c}}$ & & & & 9.44 & $0.66+/-0.04$ & & & & & \\
\hline $25-26$ & 1.20 & 0.53 & 0.56 & 9.50 & $0.63+/-0.17$ & ND & $1.12+/-0.16$ & $0.92+/-0.05$ & $1.50+/-0.19$ & $13.81+/-0.91$ \\
\hline $26-27$ & 1.22 & 0.53 & 0.56 & 8.03 & $0.63+/-0.09$ & ND & $1.03+/-0.13$ & $0.90+/-0.05$ & $1.30+/-0.15$ & $12.26+/-0.74$ \\
\hline $26-27^{\mathrm{c}}$ & & & & 8.03 & $0.58+/-0.01$ & & & & & \\
\hline $27-28$ & 1.20 & 0.53 & 0.56 & 9.17 & $0.62+/-0.05$ & ND & $1.00+/-0.16$ & $0.86+/-0.05$ & $1.21+/-0.18$ & $11.98+/-0.82$ \\
\hline $28-29$ & 1.19 & 0.53 & 0.56 & 9.10 & $0.66+/-0.04$ & ND & $1.12+/-0.16$ & $0.89+/-0.05$ & $1.51+/-0.20$ & $12.50+/-0.86$ \\
\hline $29-30$ & 1.18 & 0.51 & 0.57 & 9.16 & $0.64+/-0.02$ & ND & $1.01+/-0.16$ & $0.89+/-0.05$ & $1.48+/-0.18$ & $12.67+/-0.82$ \\
\hline $29-30^{c}$ & & & & 7.61 & $0.66+/-0.04$ & & & & & \\
\hline $30-31$ & 1.20 & 0.53 & 0.55 & 9.25 & $0.61+/-0.02$ & ND & $1.05+/-0.13$ & $0.90+/-0.05$ & $1.40+/-0.16$ & $13.22+/-0.75$ \\
\hline $31-32$ & 1.20 & 0.54 & 0.55 & 9.53 & $0.67+/-0.05$ & ND & $1.16+/-0.16$ & $0.83+/-0.05$ & $1.46+/-0.18$ & $12.48+/-0.84$ \\
\hline $32-33$ & 1.15 & 0.48 & 0.58 & 9.84 & $0.66+/-0.01$ & ND & $1.03+/-0.16$ & $0.86+/-0.05$ & $1.43+/-0.18$ & $12.23+/-0.86$ \\
\hline $33-34$ & 1.18 & 0.50 & 0.58 & 9.36 & $0.62+/-0.05$ & ND & $1.05+/-0.13$ & $0.85+/-0.04$ & $1.29+/-0.15$ & $12.42+/-0.72$ \\
\hline $34-35$ & 1.17 & 0.50 & 0.58 & 9.25 & $0.61+/-0.02$ & ND & $1.03+/-0.16$ & $0.86+/-0.05$ & $1.28+/-0.17$ & $13.81+/-0.90$ \\
\hline $35-36$ & 1.17 & 0.50 & 0.57 & 9.30 & $0.52+/-0.01$ & ND & $0.98+/-0.12$ & $0.81+/-0.04$ & $1.31+/-0.16$ & $12.85+/-0.75$ \\
\hline $36-37$ & 1.24 & 0.58 & 0.53 & 9.75 & $0.57+/-0.02$ & & & & & \\
\hline $37-38$ & 1.22 & 0.57 & 0.53 & 9.09 & $0.50+/-0.02$ & ND & $0.92+/-0.15$ & $0.80+/-0.05$ & $1.43+/-0.18$ & $12.92+/-0.83$ \\
\hline $38-39$ & 1.11 & 0.48 & 0.57 & 8.87 & $0.51+/-0.08$ & & & & & \\
\hline $39-40$ & 1.19 & 0.55 & 0.54 & 8.87 & $0.48+/-0.01$ & ND & $0.89+/-0.13$ & $0.84+/-0.04$ & $1.42+/-0.17$ & $12.87+/-0.76$ \\
\hline $40-41$ & 1.23 & 0.56 & 0.55 & 9.31 & $0.56+/-0.08$ & & & & & \\
\hline $41-42$ & 1.20 & 0.55 & 0.54 & 8.75 & $0.55+/-0.01$ & ND & $0.94+/-0.14$ & $0.80+/-0.05$ & $1.22+/-0.18$ & $12.97+/-0.85$ \\
\hline $42-43$ & 1.21 & 0.56 & 0.53 & 8.26 & $0.51+/-0.04$ & ND & $0.91+/-0.11$ & $0.80+/-0.04$ & $1.34+/-0.16$ & $12.43+/-0.71$ \\
\hline $43-44$ & 1.20 & 0.56 & 0.53 & 7.98 & $0.40+/-0.04$ & & & & & \\
\hline $43-44^{c}$ & & & & 9.81 & $0.36+/-0.03$ & & & & & \\
\hline
\end{tabular}

$\mathrm{a}=$ results determined by alpha spectroscopy method; $\mathrm{b}=$ results determined by gamma spectroscopy method; $\mathrm{c}=$ replicate analysis

$\mathrm{L}_{\mathrm{c}: \mathrm{Cs}-137}=0.02 \mathrm{pCi} / \mathrm{g} ; \mathrm{L}_{\mathrm{c}: \mathrm{Pb}-210}=0.10 \mathrm{pCi} / \mathrm{g} ; \mathrm{L}_{\mathrm{c}: \mathrm{Ra}-226}=0.05 \mathrm{pCi} / \mathrm{g} ; \mathrm{L}_{\mathrm{c}: \mathrm{Th}-234}=0.12 \mathrm{pCi} / \mathrm{g} ; \mathrm{L}_{\mathrm{c}: \mathrm{K}-40}=0.28 \mathrm{pCi} / \mathrm{g}$ 
Table 5. Physical and radioisotopic data for Mobile Bay core MB0810-7BC.

[cm, centimeter; $\mathrm{g} / \mathrm{cm}^{3}$, grams per cubic centimeter; \% Mass, percent mass; g OM $/ \mathrm{g}$ sed, grams of organic matter per grams of sediment; $\mathrm{pCi} / \mathrm{g}$, picocuries per gram; $\mathrm{ND}$, not detected; $L_{c}$, critical level]

\begin{tabular}{|c|c|c|c|c|c|c|c|c|c|c|}
\hline Depth $\quad(\mathrm{cm})$ & $\begin{array}{c}\text { Wet Bulk } \\
\text { Density } \\
\left(\mathrm{g} / \mathrm{cm}^{3}\right)\end{array}$ & $\begin{array}{c}\text { Dry Bulk } \\
\text { Density } \\
\left(\mathrm{g} / \mathrm{cm}^{3}\right)\end{array}$ & $\begin{array}{c}\text { Water } \\
\text { Content } \\
\text { (\% Mass) }\end{array}$ & $\begin{array}{l}\text { Loss On } \\
\text { Ignition (g } \\
\text { OM/g sed) }\end{array}$ & $\mathrm{Pb}-210^{\mathrm{a}} \quad(\mathrm{pCi} / \mathrm{g})$ & $\begin{array}{l}\mathrm{Cs}-137^{\mathrm{b}} \\
(\mathrm{pCi} / \mathrm{g})\end{array}$ & $\begin{array}{l}\mathrm{Pb}-210^{\mathrm{b}} \\
(\mathrm{pCi} / \mathrm{g})\end{array}$ & $\begin{array}{l}\mathrm{Ra}-226^{\mathrm{b}} \\
(\mathrm{pCi} / \mathrm{g})\end{array}$ & Th-234 $\quad$ (pCi/g) & $\begin{array}{l}\mathrm{K}-40^{\mathrm{b}} \\
(\mathrm{pCi} / \mathrm{g})\end{array}$ \\
\hline $0-1$ & 1.11 & 0.41 & 0.63 & 10.64 & $2.19+/-0.02$ & $0.10+/-0.02$ & $2.85+/-0.15$ & $0.71+/-0.04$ & $1.00+/-0.15$ & $11.20+/-0.73$ \\
\hline $1-2$ & 1.12 & 0.43 & 0.61 & 10.29 & $2.37+/-0.05$ & $0.11+/-0.03$ & $2.66+/-0.19$ & $0.64+/-0.05$ & $0.89+/-0.16$ & $11.75+/-0.86$ \\
\hline $2-3$ & 1.12 & 0.49 & 0.56 & 9.84 & $2.03+/-0.09$ & $0.12+/-0.03$ & $2.55+/-0.18$ & $0.64+/-0.05$ & $0.92+/-0.16$ & $10.98+/-0.83$ \\
\hline $3-4$ & 1.16 & 0.45 & 0.61 & 8.94 & $1.96+/-0.08$ & $0.09+/-0.02$ & $2.54+/-0.15$ & $0.70+/-0.04$ & $1.05+/-0.14$ & $10.19+/-0.69$ \\
\hline $4-5$ & 1.15 & 0.47 & 0.59 & 9.06 & $1.81+/-0.12$ & $0.09+/-0.02$ & $2.54+/-0.15$ & $0.67+/-0.04$ & $1.06+/-0.13$ & $10.30+/-0.68$ \\
\hline $4-5^{c}$ & & & & 7.76 & $1.91+/-0.25$ & & & & & \\
\hline $5-6$ & 1.15 & 0.46 & 0.61 & 9.03 & $1.89+/-0.08$ & $0.10+/-0.02$ & $2.38+/-0.12$ & $0.64+/-0.03$ & $0.91+/-0.11$ & $10.72+/-0.57$ \\
\hline $6-7$ & 1.19 & 0.50 & 0.58 & 9.18 & $2.01+/-0.04$ & $0.11+/-0.02$ & $2.61+/-0.15$ & $0.65+/-0.04$ & $1.07+/-0.13$ & $10.33+/-0.68$ \\
\hline $7-8$ & 1.08 & 0.38 & 0.65 & 9.38 & $1.99+/-0.03$ & $0.09+/-0.04$ & $2.70+/-0.23$ & $0.63+/-0.06$ & $1.01+/-0.19$ & $10.61+/-1.04$ \\
\hline $8-9$ & 1.13 & 0.44 & 0.61 & 9.81 & $2.01+/-0.06$ & $0.10+/-0.02$ & $2.70+/-0.13$ & $0.71+/-0.04$ & $1.12+/-0.13$ & $11.13+/-0.61$ \\
\hline $9-10$ & 1.15 & 0.48 & 0.58 & 9.84 & $1.98+/-0.01$ & $0.11+/-0.03$ & $2.66+/-0.18$ & $0.74+/-0.05$ & $1.12+/-0.16$ & $10.65+/-0.8$ \\
\hline $10-11$ & 1.19 & 0.49 & 0.59 & 8.55 & $1.77+/-0.01$ & $0.11+/-0.02$ & $2.43+/-0.15$ & $0.61+/-0.04$ & $0.94+/-0.13$ & $10.56+/-0.66$ \\
\hline $11-12$ & 1.18 & 0.48 & 0.59 & 9.19 & $1.95+/-0.15$ & $0.12+/-0.03$ & $2.33+/-0.16$ & $0.63+/-0.05$ & $0.97+/-0.15$ & $10.38+/-0.82$ \\
\hline $12-13$ & 1.18 & 0.48 & 0.59 & 8.95 & $1.70+/-0.07$ & $0.10+/-0.02$ & $2.39+/-0.15$ & $0.66+/-0.04$ & $1.11+/-0.14$ & $9.68+/-0.69$ \\
\hline $13-14$ & 1.19 & 0.49 & 0.59 & 9.56 & $1.78+/-0.10$ & $0.09+/-0.02$ & $2.41+/-0.12$ & $0.66+/-0.03$ & $1.14+/-0.12$ & $10.93+/-0.59$ \\
\hline $14-15$ & 1.16 & 0.48 & 0.59 & 8.48 & $1.72+/-0.02$ & $0.09+/-0.03$ & $2.31+/-0.19$ & $0.67+/-0.05$ & $1.07+/-0.16$ & $10.16+/-0.81$ \\
\hline $15-16$ & 1.20 & 0.51 & 0.57 & 7.66 & $1.48+/-0.08$ & $0.08+/-0.02$ & $2.09+/-0.12$ & $0.66+/-0.04$ & $0.96+/-0.11$ & $9.50+/-0.57$ \\
\hline $16-17$ & 1.17 & 0.52 & 0.55 & 7.61 & $1.68+/-0.13$ & $0.08+/-0.03$ & $2.28+/-0.17$ & $0.64+/-0.05$ & $0.97+/-0.14$ & $10.07+/-0.77$ \\
\hline $16-17^{\mathrm{c}}$ & & & & 6.68 & $1.49+/-0.16$ & & & & & \\
\hline $17-18$ & 1.20 & 0.52 & 0.57 & 7.99 & $1.56+/-0.05$ & $0.09+/-0.03$ & $1.98+/-0.17$ & $0.62+/-0.05$ & $1.11+/-0.16$ & $9.74+/-0.78$ \\
\hline $18-19$ & 1.28 & 0.60 & 0.53 & 7.75 & $1.58+/-0.01$ & $0.10+/-0.02$ & $2.16+/-0.14$ & $0.65+/-0.04$ & $1.04+/-0.14$ & $9.26+/-0.65$ \\
\hline $19-20$ & 1.20 & 0.51 & 0.57 & 7.15 & $1.72+/-0.06$ & $0.09+/-0.03$ & $1.99+/-0.17$ & $0.62+/-0.05$ & $0.91+/-0.16$ & $9.78+/-0.79$ \\
\hline $20-21$ & 1.23 & 0.59 & 0.52 & 6.69 & $1.54+/-0.02$ & $0.09+/-0.03$ & $2.06+/-0.17$ & $0.65+/-0.05$ & $0.95+/-0.16$ & $9.37+/-0.76$ \\
\hline $20-21^{\mathrm{c}}$ & & & & 6.20 & $1.43+/-0.05$ & & & & & \\
\hline $21-22$ & 1.21 & 0.58 & 0.52 & 7.82 & $1.54+/-0.05$ & $0.09+/-0.03$ & $2.00+/-0.14$ & $0.61+/-0.04$ & $0.98+/-0.13$ & $9.66+/-0.67$ \\
\hline $22-23$ & 1.28 & 0.65 & 0.49 & 8.25 & $1.55+/-0.02$ & $0.08+/-0.03$ & $2.07+/-0.16$ & $0.61+/-0.05$ & $1.14+/-0.16$ & $9.60+/-0.76$ \\
\hline $23-24$ & 1.25 & 0.64 & 0.49 & 7.10 & $1.42+/-0.02$ & $0.06+/-0.03$ & $2.01+/-0.16$ & $0.67+/-0.05$ & $1.15+/-0.16$ & $9.96+/-0.78$ \\
\hline $24-25$ & 1.31 & 0.69 & 0.48 & 6.75 & $1.27+/-0.05$ & $0.07+/-0.02$ & $1.87+/-0.14$ & $0.60+/-0.04$ & $1.12+/-0.14$ & $9.73+/-0.68$ \\
\hline $25-26$ & 1.26 & 0.63 & 0.50 & 6.44 & $1.09+/-0.06$ & $0.05+/-0.03$ & $1.64+/-0.17$ & $0.62+/-0.05$ & $1.16+/-0.17$ & $9.49+/-0.78$ \\
\hline $26-27$ & 1.28 & 0.63 & 0.51 & 7.52 & $0.98+/-0.03$ & $0.08+/-0.03$ & $1.50+/-0.15$ & $0.67+/-0.05$ & $1.13+/-0.16$ & $8.27+/-0.72$ \\
\hline $26-27^{\mathrm{c}}$ & & & & 5.67 & $0.96+/-0.01$ & & & & & \\
\hline
\end{tabular}


Table 6. Physical and radioisotopic data for Mobile Bay core MB0810-8BC.

[cm, centimeter; $\mathrm{g} / \mathrm{cm}^{3}$, grams per cubic centimeter; \% Mass, percent mass; g OM/g sed, grams of organic matter per grams of sediment; $\mathrm{pCi} / \mathrm{g}$, picocuries per gram; ND, not detected; $\mathrm{L}_{\mathrm{c}}$, critical level]

\begin{tabular}{|c|c|c|c|c|c|c|c|c|c|c|}
\hline Depth $\quad(\mathrm{cm})$ & $\begin{array}{c}\text { Wet Bulk } \\
\text { Density } \\
\left(\mathrm{g} / \mathrm{cm}^{3}\right)\end{array}$ & $\begin{array}{c}\text { Dry Bulk } \\
\text { Density } \\
\left(\mathrm{g} / \mathrm{cm}^{3}\right)\end{array}$ & $\begin{array}{c}\text { Water } \\
\text { Content } \\
\text { (\% Mass) }\end{array}$ & $\begin{array}{l}\text { Loss On } \\
\text { Ignition } \\
\text { OM/g sed) }\end{array}$ & $\mathrm{Pb}-210^{\mathrm{a}} \quad(\mathrm{pCi} / \mathrm{g})$ & $\begin{array}{c}\text { Cs-137 } \\
\text { (pCi/g) }\end{array}$ & $\begin{array}{l}\mathrm{Pb}-210^{\mathrm{b}} \\
(\mathrm{pCi} / \mathrm{g})\end{array}$ & $\begin{array}{l}\text { Ra-226 } \\
\text { (pCi/g) }\end{array}$ & Th-234 ${ }^{b} \quad(p C i / g)$ & $\begin{array}{l}\mathrm{K}-40^{\mathrm{b}} \\
(\mathrm{pCi} / \mathrm{g})\end{array}$ \\
\hline $0-1$ & 1.22 & 0.46 & 0.62 & 14.48 & $3.16+/-0.06$ & $0.12+/-0.02$ & $3.91+/-0.17$ & $0.67+/-0.04$ & $1.05+/-0.14$ & $10.44+/-0.66$ \\
\hline $1-2$ & 1.22 & 0.44 & 0.64 & 14.28 & $3.37+/-0.13$ & $0.13+/-0.03$ & $3.76+/-0.16$ & $0.64+/-0.04$ & $0.99+/-0.14$ & $11.16+/-0.68$ \\
\hline $1-2^{c}$ & & & & 12.57 & $2.98+/-0.03$ & & & & & \\
\hline $2-3$ & 1.22 & 0.45 & 0.63 & 14.61 & $3.41+/-0.03$ & $0.11+/-0.03$ & $3.99+/-0.20$ & $0.61+/-0.05$ & $1.06+/-0.16$ & $9.97+/-0.75$ \\
\hline $3-4$ & 1.15 & 0.36 & 0.68 & 15.02 & $3.05+/-0.08$ & $0.13+/-0.04$ & $4.18+/-0.29$ & $0.68+/-0.06$ & $0.95+/-0.23$ & $12.39+/-1.13$ \\
\hline $4-5$ & 1.19 & 0.34 & 0.71 & 14.04 & $3.29+/-0.01$ & $0.12+/-0.02$ & $3.61+/-0.15$ & $0.68+/-0.04$ & $0.97+/-0.12$ & $10.61+/-0.59$ \\
\hline $5-6$ & 1.23 & 0.39 & 0.68 & 13.13 & $3.03+/-0.09$ & $0.14+/-0.03$ & $3.57+/-0.20$ & $0.66+/-0.05$ & $1.02+/-0.16$ & $10.94+/-0.80$ \\
\hline $6-7$ & 1.19 & 0.38 & 0.69 & 12.52 & $3.11+/-0.19$ & $0.10+/-0.02$ & $3.40+/-0.15$ & $0.66+/-0.04$ & $1.08+/-0.12$ & $10.97+/-0.64$ \\
\hline $7-8$ & 1.23 & 0.44 & 0.65 & 12.48 & $2.79+/-0.09$ & $0.12+/-0.03$ & $3.26+/-0.20$ & $0.65+/-0.05$ & $1.07+/-0.16$ & $10.23+/-0.79$ \\
\hline $8-9$ & 1.21 & 0.43 & 0.64 & 13.36 & $2.74+/-0.11$ & $0.13+/-0.03$ & $3.18+/-0.17$ & $0.70+/-0.05$ & $1.06+/-0.14$ & $12.25+/-0.88$ \\
\hline $9-10$ & 1.24 & 0.44 & 0.64 & 11.41 & $2.45+/-0.06$ & $0.08+/-0.02$ & $3.21+/-0.14$ & $0.69+/-0.03$ & $1.05+/-0.11$ & $9.63+/-0.54$ \\
\hline $10-11$ & 1.25 & 0.47 & 0.62 & 9.07 & $2.29+/-0.08$ & $0.10+/-0.03$ & $3.21+/-0.19$ & $0.75+/-0.05$ & $1.07+/-0.15$ & $8.82+/-0.74$ \\
\hline $11-12$ & 1.26 & 0.48 & 0.62 & 8.71 & $2.41+/-0.12$ & $0.10+/-0.02$ & $3.06+/-0.15$ & $0.71+/-0.04$ & $1.05+/-0.12$ & $9.83+/-0.66$ \\
\hline $11-12^{\mathrm{c}}$ & & & & 8.89 & $2.17+/-0.01$ & & & & & \\
\hline $12-13$ & 1.28 & 0.50 & 0.61 & 9.41 & $2.61+/-0.18$ & $0.08+/-0.03$ & $3.22+/-0.18$ & $0.67+/-0.05$ & $1.15+/-0.16$ & $9.08+/-0.73$ \\
\hline $13-14$ & 1.26 & 0.48 & 0.62 & 8.04 & $2.19+/-0.34$ & $0.10+/-0.03$ & $3.08+/-0.19$ & $0.71+/-0.05$ & $1.08+/-0.15$ & $9.62+/-0.74$ \\
\hline $14-15$ & 1.29 & 0.54 & 0.58 & 8.31 & $2.13+/-0.07$ & $0.09+/-0.03$ & $2.77+/-0.17$ & $0.70+/-0.05$ & $1.10+/-0.15$ & $10.55+/-0.83$ \\
\hline $14-15^{\mathrm{c}}$ & & & & 8.33 & $1.95+/-0.15$ & & & & & \\
\hline $15-16$ & 1.30 & 0.56 & 0.57 & 8.16 & $2.01+/-0.19$ & $0.06+/-0.03$ & $2.78+/-0.18$ & $0.64+/-0.05$ & $1.09+/-0.15$ & $9.36+/-0.76$ \\
\hline $16-17$ & 1.33 & 0.57 & 0.57 & 8.05 & $2.10+/-0.06$ & $0.09+/-0.03$ & $2.75+/-0.19$ & $0.70+/-0.05$ & $1.12+/-0.15$ & $8.71+/-0.72$ \\
\hline $17-18$ & 1.28 & 0.52 & 0.59 & 7.47 & $1.76+/-0.12$ & $0.06+/-0.03$ & $2.70+/-0.18$ & $0.68+/-0.05$ & $1.01+/-0.16$ & $8.80+/-0.70$ \\
\hline 18-19 & 1.30 & 0.58 & 0.55 & 6.30 & $1.69+/-0.03$ & $0.08+/-0.03$ & $2.41+/-0.18$ & $0.71+/-0.05$ & $1.15+/-0.17$ & $8.28+/-0.72$ \\
\hline $19-20$ & 1.31 & 0.59 & 0.55 & 5.96 & $1.45+/-0.02$ & $0.09+/-0.03$ & $2.04+/-0.15$ & $0.67+/-0.04$ & $1.11+/-0.14$ & $9.00+/-0.64$ \\
\hline $20-21$ & 1.29 & 0.57 & 0.56 & 5.93 & $1.28+/-0.04$ & $0.05+/-0.02$ & $1.83+/-0.15$ & $0.73+/-0.05$ & $1.19+/-0.16$ & $9.13+/-0.70$ \\
\hline $21-22$ & 1.30 & 0.59 & 0.54 & 8.82 & $1.24+/-0.04$ & $0.06+/-0.03$ & $1.79+/-0.18$ & $0.74+/-0.05$ & $1.24+/-0.17$ & $8.84+/-0.75$ \\
\hline $22-23$ & 1.34 & 0.61 & 0.54 & 7.63 & $1.13+/-0.04$ & $0.04+/-0.02$ & $1.73+/-0.15$ & $0.73+/-0.04$ & $1.26+/-0.15$ & $9.22+/-0.66$ \\
\hline $22-23^{\mathrm{c}}$ & & & & 7.77 & $0.99+/-0.05$ & & & & & \\
\hline $23-24$ & 1.38 & 0.64 & 0.54 & 8.99 & $1.00+/-0.07$ & ND & $1.43+/-0.16$ & $0.72+/-0.05$ & $1.10+/-0.15$ & $9.30+/-0.73$ \\
\hline $24-25$ & 1.36 & 0.62 & 0.54 & 8.95 & $1.08+/-0.05$ & ND & $1.56+/-0.16$ & $0.80+/-0.05$ & $1.20+/-0.19$ & $10.00+/-0.78$ \\
\hline $25-26$ & 1.32 & 0.60 & 0.55 & 8.70 & $1.06+/-0.01$ & & & & & \\
\hline $26-27$ & 1.30 & 0.58 & 0.56 & 8.73 & $1.06+/-0.07$ & $0.03+/-0.02$ & $1.69+/-0.14$ & $0.72+/-0.04$ & $1.10+/-0.14$ & $10.02+/-0.64$ \\
\hline $27-28$ & 1.32 & 0.61 & 0.54 & 9.94 & $1.03+/-0.06$ & & & & & \\
\hline $28-29$ & 1.31 & 0.56 & 0.58 & 10.35 & $1.08+/-0.12$ & ND & $1.55+/-0.17$ & $0.75+/-0.05$ & $1.34+/-0.18$ & $12.00+/-0.83$ \\
\hline $29-30$ & 1.34 & 0.60 & 0.55 & 10.05 & $1.07+/-0.01$ & ND & $1.53+/-0.16$ & $0.82+/-0.05$ & $1.23+/-0.16$ & $11.52+/-0.77$ \\
\hline $30-31$ & 1.28 & 0.55 & 0.57 & 10.23 & $1.32+/-0.05$ & $0.03+/-0.02$ & $1.76+/-0.12$ & $0.82+/-0.04$ & $1.34+/-0.13$ & $11.83+/-0.61$ \\
\hline $31-32$ & 1.28 & 0.57 & 0.56 & 10.68 & $1.29+/-0.03$ & ND & $1.91+/-0.18$ & $0.78+/-0.05$ & $1.24+/-0.18$ & $12.06+/-0.84$ \\
\hline $32-33$ & 1.33 & 0.62 & 0.53 & 10.68 & $1.55+/-0.20$ & $0.04+/-0.02$ & $1.96+/-0.15$ & $0.77+/-0.04$ & $1.38+/-0.15$ & $11.60+/-0.69$ \\
\hline $33-34$ & 1.30 & 0.61 & 0.53 & 10.17 & $1.30+/-0.12$ & ND & $1.56+/-0.15$ & $0.82+/-0.05$ & $1.33+/-0.15$ & $12.14+/-0.74$ \\
\hline $34-35$ & 1.32 & 0.60 & 0.55 & 10.27 & $0.86+/-0.04$ & ND & $1.41+/-0.16$ & $0.99+/-0.05$ & $1.38+/-0.17$ & $15.66+/-0.94$ \\
\hline $35-36$ & 1.33 & 0.60 & 0.55 & 10.71 & $0.88+/-0.04$ & ND & $1.28+/-0.14$ & $0.92+/-0.05$ & $1.35+/-0.15$ & $12.68+/-0.71$ \\
\hline $35-36^{\mathrm{C}}$ & & & & 10.05 & $0.80+/-0.04$ & & & & & \\
\hline $36-37$ & 1.33 & 0.60 & 0.55 & 10.10 & $0.87+/-0.01$ & ND & $1.37+/-0.15$ & $0.87+/-0.05$ & $1.36+/-0.16$ & $11.69+/-0.73$ \\
\hline $37-38$ & 1.31 & 0.58 & 0.56 & 10.78 & $0.87+/-0.04$ & ND & $1.27+/-0.11$ & $0.84+/-0.04$ & $1.54+/-0.13$ & $12.26+/-0.60$ \\
\hline $38-39$ & 1.32 & 0.57 & 0.57 & 10.88 & $0.84+/-0.06$ & ND & $1.32+/-0.18$ & $0.87+/-0.05$ & $1.39+/-0.18$ & $11.63+/-0.77$ \\
\hline $39-40$ & 1.30 & 0.59 & 0.55 & 10.09 & $0.92+/-0.04$ & ND & $1.40+/-0.13$ & $0.81+/-0.04$ & $1.23+/-0.12$ & $10.79+/-0.60$ \\
\hline $40-41$ & 1.33 & 0.59 & 0.56 & 9.44 & $0.96+/-0.01$ & ND & $1.40+/-0.16$ & $0.89+/-0.05$ & $1.39+/-0.18$ & $12.35+/-0.85$ \\
\hline $41-42$ & 1.35 & 0.63 & 0.54 & 10.79 & $0.93+/-0.04$ & & & & & \\
\hline $42-43$ & 1.39 & 0.68 & 0.51 & 10.24 & $0.96+/-0.01$ & ND & $1.31+/-0.15$ & $0.91+/-0.05$ & $1.31+/-0.17$ & $10.77+/-0.77$ \\
\hline $43-44$ & 1.33 & 0.64 & 0.52 & 9.11 & $0.83+/-0.02$ & & & & & \\
\hline $44-45$ & 1.33 & 0.65 & 0.51 & 9.23 & $0.72+/-0.04$ & ND & $1.15+/-0.15$ & $0.82+/-0.05$ & $1.34+/-0.18$ & $11.53+/-0.83$ \\
\hline $45-46$ & 1.34 & 0.65 & 0.51 & 9.62 & $0.82+/-0.02$ & & & & & \\
\hline $46-47$ & 1.36 & 0.65 & 0.52 & 9.62 & $0.77+/-0.03$ & ND & $1.16+/-0.12$ & $0.82+/-0.04$ & $1.41+/-0.14$ & $11.35+/-0.60$ \\
\hline $47-48$ & 1.37 & 0.68 & 0.50 & 8.94 & $0.70+/-0.03$ & & & & & \\
\hline $48-49$ & 1.28 & 0.59 & 0.54 & 9.98 & $0.87+/-0.03$ & ND & $1.25+/-0.15$ & $0.87+/-0.05$ & $1.24+/-0.17$ & $12.32+/-0.81$ \\
\hline
\end{tabular}

$\mathrm{a}=$ results determined by alpha spectroscopy method; $\mathrm{b}=$ results determined by gamma spectroscopy method; $\mathrm{c}=$ replicate analysis

$\mathrm{L}_{\mathrm{c}: \mathrm{Cs}-137}=0.03 \mathrm{pCi} / \mathrm{g} ; \mathrm{L}_{\mathrm{c}: \mathrm{Pb}-210}=0.13 \mathrm{pCi} / \mathrm{g} ; \mathrm{L}_{\mathrm{c}: \mathrm{Ra}-226}=0.07 \mathrm{pCi} / \mathrm{g} ; \mathrm{L}_{\mathrm{c}: \mathrm{Th}-234}=0.15 \mathrm{pCi} / \mathrm{g} ; \mathrm{L}_{\mathrm{c}: \mathrm{K}-40}=0.38 \mathrm{pCi} / \mathrm{g}$ 
Table 7. Physical and radioisotopic data for Mobile Bay core MB0810-12BC.

[cm, centimeter; $\mathrm{g} / \mathrm{cm}^{3}$, grams per cubic centimeter; \% Mass, percent mass; g OM/g sed, grams of organic matter per grams of sediment; $\mathrm{pCi} / \mathrm{g}$, picocuries per gram; ND, not detected; $\mathrm{L}_{c}$, critical level]

\begin{tabular}{|c|c|c|c|c|c|c|c|c|c|c|}
\hline Depth $\quad(\mathrm{cm})$ & $\begin{array}{c}\text { Wet Bulk } \\
\text { Density } \\
\left(\mathrm{g} / \mathrm{cm}^{3}\right)\end{array}$ & $\begin{array}{c}\text { Dry Bulk } \\
\text { Density } \\
\left(\mathrm{g} / \mathrm{cm}^{3}\right)\end{array}$ & $\begin{array}{c}\text { Water } \\
\text { Content } \\
\text { (\% Mass) }\end{array}$ & $\begin{array}{l}\text { Loss On } \\
\text { Ignition } \\
\text { OM/g sed) }\end{array}$ & $\mathrm{Pb}-210^{\mathrm{a}} \quad(\mathrm{pCi} / \mathrm{g})$ & $\begin{array}{l}\text { Cs-137 } \\
(\mathrm{pCi} / \mathrm{g})\end{array}$ & $\begin{array}{r}\mathrm{Pb}-210^{\mathrm{b}} \\
(\mathrm{pCi} / \mathrm{g})\end{array}$ & $\begin{array}{r}\mathrm{Ra}-226^{\mathrm{b}} \\
\text { (pCi/g) }\end{array}$ & Th-234 ${ }^{\mathrm{b}} \quad(\mathrm{pCi} / \mathrm{g})$ & $\begin{array}{l}\mathrm{K}-40^{\mathrm{b}} \\
(\mathrm{pCi} / \mathrm{g})\end{array}$ \\
\hline $0-1$ & 1.13 & 0.39 & 0.65 & 9.58 & $1.95+/-0.21$ & $0.10+/-0.02$ & $2.84+/-0.14$ & $0.77+/-0.04$ & $1.20+/-0.13$ & $10.13+/-0.57$ \\
\hline $1-2$ & 1.12 & 0.37 & 0.67 & 9.26 & $1.94+/-0.01$ & $0.08+/-0.03$ & $2.83+/-0.16$ & $0.75+/-0.04$ & $1.04+/-0.14$ & $9.93+/-0.68$ \\
\hline $2-3$ & 1.27 & 0.53 & 0.59 & 8.58 & $1.87+/-0.04$ & $0.12+/-0.05$ & $2.57+/-0.24$ & $0.78+/-0.07$ & $1.17+/-0.21$ & $9.99+/-1.05$ \\
\hline $3-4$ & 1.23 & 0.45 & 0.63 & 8.00 & $1.79+/-0.04$ & $0.07+/-0.03$ & $2.34+/-0.15$ & $0.81+/-0.05$ & $1.36+/-0.15$ & $9.35+/-0.66$ \\
\hline $3-4^{c}$ & & & & 9.39 & $1.64+/-0.01$ & & & & & \\
\hline $4-5$ & 1.30 & 0.52 & 0.60 & 8.61 & $1.44+/-0.07$ & $0.11+/-0.03$ & $1.85+/-0.17$ & $0.83+/-0.05$ & $1.55+/-0.18$ & $10.99+/-0.78$ \\
\hline $5-6$ & 1.28 & 0.53 & 0.59 & 8.49 & $1.31+/-0.03$ & $0.09+/-0.03$ & $1.84+/-0.17$ & $0.77+/-0.05$ & $1.53+/-0.18$ & $11.71+/-0.83$ \\
\hline $6-7$ & 1.35 & 0.57 & 0.58 & 9.14 & $1.17+/-0.05$ & $0.08+/-0.03$ & $1.68+/-0.14$ & $0.84+/-0.05$ & $1.62+/-0.15$ & $11.14+/-0.72$ \\
\hline $7-8$ & 1.38 & 0.59 & 0.57 & 9.20 & $1.13+/-0.04$ & $0.08+/-0.03$ & $1.55+/-0.14$ & $0.79+/-0.05$ & $1.58+/-0.16$ & $11.01+/-0.78$ \\
\hline $8-9$ & 1.31 & 0.55 & 0.58 & 9.08 & $1.13+/-0.05$ & $0.07+/-0.02$ & $1.61+/-0.11$ & $0.74+/-0.03$ & $1.64+/-0.12$ & $11.38+/-0.54$ \\
\hline $9-10$ & 1.40 & 0.61 & 0.57 & 8.76 & $1.10+/-0.08$ & $0.06+/-0.03$ & $1.73+/-0.15$ & $0.74+/-0.05$ & $1.59+/-0.17$ & $11.93+/-0.82$ \\
\hline $10-11$ & 1.30 & 0.55 & 0.58 & 9.43 & $1.03+/-0.11$ & $0.06+/-0.03$ & $1.43+/-0.16$ & $0.73+/-0.05$ & $1.55+/-0.17$ & $10.54+/-0.81$ \\
\hline $11-12$ & 1.33 & 0.56 & 0.58 & 9.51 & $1.12+/-0.06$ & $0.06+/-0.03$ & $1.57+/-0.14$ & $0.79+/-0.05$ & $1.73+/-0.16$ & $11.58+/-0.72$ \\
\hline $12-13$ & 1.37 & 0.59 & 0.57 & 9.16 & $0.93+/-0.01$ & $0.06+/-0.03$ & $1.44+/-0.15$ & $0.79+/-0.05$ & $1.50+/-0.16$ & $11.38+/-0.78$ \\
\hline $13-14$ & 1.29 & 0.53 & 0.59 & 8.72 & $0.88+/-0.01$ & $0.05+/-0.02$ & $1.43+/-0.14$ & $0.79+/-0.04$ & $1.70+/-0.16$ & $11.82+/-0.70$ \\
\hline $13-14^{\mathrm{c}}$ & & & & 9.39 & $0.84+/-0.01$ & & & & & \\
\hline $14-15$ & 1.27 & 0.50 & 0.61 & 9.21 & $0.98+/-0.05$ & $0.03+/-0.03$ & $1.29+/-0.16$ & $0.85+/-0.05$ & $1.42+/-0.17$ & $12.40+/-0.82$ \\
\hline $15-16$ & 1.29 & 0.57 & 0.56 & 8.91 & $0.83+/-0.01$ & ND & $1.33+/-0.16$ & $0.83+/-0.05$ & $1.51+/-0.19$ & $11.73+/-0.84$ \\
\hline $16-17$ & 1.28 & 0.51 & 0.60 & 8.74 & $0.91+/-0.04$ & ND & $1.51+/-0.17$ & $0.82+/-0.05$ & $1.47+/-0.17$ & $12.26+/-0.86$ \\
\hline $17-18$ & 1.29 & 0.53 & 0.59 & 9.05 & $0.86+/-0.01$ & ND & $1.38+/-0.15$ & $0.82+/-0.04$ & $1.47+/-0.17$ & $12.21+/-0.74$ \\
\hline $18-19$ & 1.29 & 0.53 & 0.59 & 8.67 & $0.77+/-0.01$ & ND & $1.23+/-0.15$ & $0.78+/-0.05$ & $1.45+/-0.19$ & $12.78+/-0.82$ \\
\hline $19-20$ & 1.22 & 0.50 & 0.59 & 8.18 & $0.78+/-0.10$ & ND & $1.23+/-0.16$ & $0.85+/-0.05$ & $1.41+/-0.19$ & $11.37+/-0.83$ \\
\hline $19-20^{c}$ & & & & 9.39 & $0.79+/-0.02$ & & & & & \\
\hline $20-21$ & 1.30 & 0.56 & 0.57 & 8.23 & $0.80+/-0.01$ & ND & $1.32+/-0.13$ & $0.84+/-0.05$ & $1.48+/-0.15$ & $11.63+/-0.72$ \\
\hline $21-22$ & 1.24 & 0.49 & 0.60 & 8.01 & $0.82+/-0.02$ & ND & $1.30+/-0.15$ & $0.86+/-0.05$ & $1.45+/-0.17$ & $12.53+/-0.84$ \\
\hline $22-23$ & 1.38 & 0.64 & 0.54 & 7.60 & $0.77+/-0.04$ & ND & $1.29+/-0.15$ & $0.91+/-0.05$ & $1.46+/-0.17$ & $12.30+/-0.85$ \\
\hline $23-24$ & 1.35 & 0.58 & 0.57 & 7.75 & $0.70+/-0.02$ & ND & $1.25+/-0.14$ & $0.88+/-0.04$ & $1.44+/-0.14$ & $12.08+/-0.73$ \\
\hline $24-25$ & 1.33 & 0.57 & 0.57 & 7.44 & $0.72+/-0.01$ & ND & $1.33+/-0.16$ & $0.93+/-0.05$ & $1.40+/-0.18$ & $10.85+/-0.82$ \\
\hline $25-26$ & 1.30 & 0.54 & 0.58 & 9.98 & $0.79+/-0.09$ & ND & $1.26+/-0.13$ & $0.87+/-0.04$ & $1.35+/-0.14$ & $12.12+/-0.70$ \\
\hline $26-27$ & 1.34 & 0.59 & 0.56 & 9.88 & $0.76+/-0.01$ & ND & $1.22+/-0.13$ & $0.87+/-0.04$ & $1.31+/-0.14$ & $11.80+/-0.68$ \\
\hline $27-28$ & 1.33 & 0.56 & 0.57 & 9.80 & $0.85+/-0.01$ & ND & $1.28+/-0.14$ & $0.84+/-0.04$ & $1.37+/-0.14$ & $12.09+/-0.69$ \\
\hline $27-28^{\mathrm{c}}$ & & & & 9.39 & $0.75+/-0.01$ & & & & & \\
\hline $28-29$ & 1.35 & 0.59 & 0.56 & 10.29 & $0.83+/-0.03$ & ND & $1.18+/-0.12$ & $0.88+/-0.04$ & $1.31+/-0.14$ & $12.80+/-0.64$ \\
\hline $29-30$ & 1.28 & 0.52 & 0.59 & 9.89 & $0.81+/-0.08$ & ND & $1.17+/-0.14$ & $0.84+/-0.05$ & $1.33+/-0.17$ & $12.03+/-0.80$ \\
\hline $30-31$ & 1.28 & 0.51 & 0.60 & 9.71 & $0.74+/-0.01$ & & & & & \\
\hline $31-32$ & 1.29 & 0.55 & 0.58 & 10.65 & $0.80+/-0.06$ & & & & & \\
\hline $32-33$ & 1.34 & 0.59 & 0.56 & 10.19 & $0.76+/-0.05$ & & & & & \\
\hline $33-34$ & 1.42 & 0.66 & 0.54 & 9.17 & $0.80+/-0.05$ & & & & & \\
\hline $34-35$ & 1.37 & 0.56 & 0.59 & 7.73 & $0.65+/-0.03$ & & & & & \\
\hline $35-36$ & 1.32 & 0.54 & 0.59 & 9.07 & $0.73+/-0.01$ & & & & & \\
\hline $35-36^{\mathrm{c}}$ & & & & 9.39 & $0.73+/-0.02$ & & & & & \\
\hline $36-37$ & 1.32 & 0.55 & 0.59 & 9.11 & $0.71+/-0.03$ & & & & & \\
\hline $37-38$ & 1.35 & 0.53 & 0.61 & 9.39 & $0.72+/-0.03$ & & & & & \\
\hline $38-39$ & 1.32 & 0.51 & 0.61 & & & & & & & \\
\hline
\end{tabular}

$\mathrm{a}=$ results determined by alpha spectroscopy method; $\mathrm{b}=$ results determined by gamma spectroscopy method; $\mathrm{c}=$ replicate analysis

$\mathrm{L}_{\mathrm{c}: \mathrm{Cs}-137}=0.04 \mathrm{pCi} / \mathrm{g} ; \mathrm{L}_{\mathrm{c}: \mathrm{Pb}-210}=0.11 \mathrm{pCi} / \mathrm{g} ; \mathrm{L}_{\mathrm{c}: \mathrm{Ra}-226}=0.07 \mathrm{pCi} / \mathrm{g} ; \mathrm{L}_{\mathrm{c}: \mathrm{Th}-234}=0.13 \mathrm{pCi} / \mathrm{g} ; \mathrm{L}_{\mathrm{c}: \mathrm{K}-40}=0.40 \mathrm{pCi} / \mathrm{g}$ 
Table 8. Physical and radioisotopic data for Mobile Bay core MB0810-20BC.

[cm, centimeter; $\mathrm{g} / \mathrm{cm}^{3}$, grams per cubic centimeter; \% Mass, percent mass; g OM/g sed, grams of organic matter per grams of sediment; $\mathrm{pCi} / \mathrm{g}$, picocuries per gram; ND, not detected; $\mathrm{L}_{\mathrm{c}}$, critical level]

\begin{tabular}{|c|c|c|c|c|c|c|c|c|c|c|}
\hline Depth $\quad(\mathrm{cm})$ & $\begin{array}{c}\text { Wet Bulk } \\
\text { Density } \\
\left(\mathrm{g} / \mathrm{cm}^{3}\right)\end{array}$ & $\begin{array}{c}\text { Dry Bulk } \\
\text { Density } \\
\left(\mathrm{g} / \mathrm{cm}^{3}\right)\end{array}$ & $\begin{array}{c}\text { Water } \\
\text { Content } \\
\text { (\% Mass) }\end{array}$ & $\begin{array}{l}\text { Loss On } \\
\text { Ignition } \\
\text { OM/g sed) }\end{array}$ & $\mathrm{Pb}-210^{\mathrm{a}} \quad(\mathrm{pCi} / \mathrm{g})$ & $\begin{array}{l}\mathrm{Cs}-137^{\mathrm{b}} \\
(\mathrm{pCi} / \mathrm{g})\end{array}$ & $\begin{array}{l}\mathrm{Pb}-210^{\mathrm{b}} \\
(\mathrm{pCi} / \mathrm{g})\end{array}$ & $\begin{array}{l}\mathrm{Ra}-226^{\mathrm{b}} \\
(\mathrm{pCi} / \mathrm{g})\end{array}$ & Th-234 ${ }^{b} \quad(p C i / g)$ & $\begin{array}{l}\mathrm{K}-40^{\mathrm{b}} \\
(\mathrm{pCi} / \mathrm{g})\end{array}$ \\
\hline $0-1$ & 1.05 & 0.32 & 0.70 & 12.02 & $2.49+/-0.01$ & $0.13+/-0.03$ & $3.28+/-0.15$ & $0.69+/-0.04$ & $1.04+/-0.14$ & $11.33+/-0.66$ \\
\hline $1-2$ & 1.08 & 0.36 & 0.67 & 12.11 & $2.50+/-0.01$ & $0.12+/-0.02$ & $3.36+/-0.14$ & $0.68+/-0.04$ & $1.07+/-0.12$ & $11.62+/-0.61$ \\
\hline $2-3$ & 1.10 & 0.34 & 0.69 & 11.16 & $2.51+/-0.28$ & $0.12+/-0.04$ & $3.26+/-0.21$ & $0.69+/-0.06$ & $1.07+/-0.17$ & $13.79+/-1.07$ \\
\hline $3-4$ & 1.07 & 0.33 & 0.69 & 11.29 & $2.53+/-0.11$ & $0.12+/-0.04$ & $3.43+/-0.23$ & $0.72+/-0.06$ & $1.11+/-0.19$ & $13.25+/-1.13$ \\
\hline $4-5$ & 1.07 & 0.34 & 0.68 & 12.36 & $2.33+/-0.15$ & $0.12+/-0.03$ & $3.29+/-0.19$ & $0.75+/-0.05$ & $1.25+/-0.17$ & $12.64+/-0.99$ \\
\hline $5-6$ & 1.08 & 0.35 & 0.68 & 12.28 & $2.67+/-0.06$ & $0.11+/-0.03$ & $3.36+/-0.16$ & $0.74+/-0.05$ & $1.18+/-0.15$ & $13.71+/-0.89$ \\
\hline $6-7$ & 1.04 & 0.33 & 0.68 & 12.42 & $2.58+/-0.55$ & $0.14+/-0.03$ & $3.16+/-0.16$ & $0.69+/-0.05$ & $1.32+/-0.17$ & $13.71+/-0.90$ \\
\hline $7-8$ & 1.10 & 0.42 & 0.62 & 11.22 & $2.79+/-0.05$ & $0.16+/-0.03$ & $3.16+/-0.18$ & $0.70+/-0.05$ & $1.32+/-0.15$ & $11.77+/-0.79$ \\
\hline $8-9$ & 1.09 & 0.39 & 0.65 & 10.89 & $2.69+/-0.20$ & $0.13+/-0.03$ & $3.44+/-0.20$ & $0.66+/-0.05$ & $1.12+/-0.17$ & $11.72+/-0.86$ \\
\hline $9-10$ & 1.11 & 0.40 & 0.64 & 10.70 & $2.47+/-0.15$ & $0.12+/-0.03$ & $3.14+/-0.17$ & $0.70+/-0.04$ & $1.35+/-0.15$ & $11.77+/-0.74$ \\
\hline $10-11$ & 1.12 & 0.42 & 0.62 & 10.30 & $2.32+/-0.04$ & $0.12+/-0.03$ & $3.10+/-0.18$ & $0.71+/-0.05$ & $1.30+/-0.17$ & $11.45+/-0.79$ \\
\hline $10-11^{\mathrm{c}}$ & & & & 8.94 & $2.24+/-0.07$ & & & & & \\
\hline $11-12$ & 1.12 & 0.41 & 0.64 & 10.08 & $2.08+/-0.06$ & $0.12+/-0.03$ & $2.68+/-0.18$ & $0.68+/-0.05$ & $1.38+/-0.17$ & $11.64+/-0.86$ \\
\hline $12-13$ & 1.11 & 0.43 & 0.61 & 10.43 & $2.55+/-0.04$ & $0.16+/-0.03$ & $2.95+/-0.15$ & $0.72+/-0.04$ & $1.43+/-0.13$ & $11.21+/-0.69$ \\
\hline $13-14$ & 1.14 & 0.46 & 0.60 & 10.65 & $2.21+/-0.05$ & $0.15+/-0.03$ & $2.65+/-0.16$ & $0.68+/-0.04$ & $1.63+/-0.15$ & $11.81+/-0.73$ \\
\hline $13-14^{c}$ & & & & 8.83 & $2.14+/-0.37$ & & & & & \\
\hline $14-15$ & 1.14 & 0.46 & 0.60 & 10.37 & $1.91+/-0.23$ & $0.13+/-0.03$ & $2.65+/-0.18$ & $0.69+/-0.05$ & $1.65+/-0.17$ & $11.60+/-0.80$ \\
\hline $15-16$ & 1.19 & 0.48 & 0.59 & 9.98 & $1.86+/-0.12$ & $0.16+/-0.04$ & $2.45+/-0.17$ & $0.69+/-0.05$ & $1.61+/-0.17$ & $12.31+/-0.87$ \\
\hline $16-17$ & 1.19 & 0.49 & 0.59 & 10.09 & $1.87+/-0.08$ & $0.16+/-0.03$ & $2.44+/-0.15$ & $0.68+/-0.04$ & $1.70+/-0.15$ & $12.24+/-0.70$ \\
\hline $17-18$ & 1.14 & 0.45 & 0.60 & 10.53 & $1.76+/-0.06$ & $0.14+/-0.03$ & $2.22+/-0.13$ & $0.67+/-0.04$ & $1.83+/-0.14$ & $11.94+/-0.62$ \\
\hline $18-19$ & 1.15 & 0.47 & 0.59 & 10.42 & $1.51+/-0.11$ & $0.14+/-0.03$ & $2.09+/-0.16$ & $0.70+/-0.05$ & $1.86+/-0.18$ & $12.09+/-0.81$ \\
\hline $19-20$ & 1.14 & 0.47 & 0.59 & 9.69 & $1.46+/-0.02$ & $0.13+/-0.03$ & $1.81+/-0.15$ & $0.72+/-0.04$ & $1.97+/-0.16$ & $13.00+/-0.74$ \\
\hline $20-21$ & 1.18 & 0.49 & 0.59 & 9.09 & $1.57+/-0.06$ & $0.13+/-0.03$ & $1.71+/-0.17$ & $0.72+/-0.05$ & $1.76+/-0.19$ & $13.00+/-0.85$ \\
\hline $20-21^{\mathrm{c}}$ & & & & 8.29 & $1.28+/-0.03$ & & & & & \\
\hline $20-21^{\mathrm{c}}$ & & & & 10.16 & $1.42+/-0.12$ & & & & & \\
\hline $21-22$ & 1.13 & 0.47 & 0.59 & 9.68 & $1.62+/-0.08$ & $0.21+/-0.04$ & $1.83+/-0.17$ & $0.68+/-0.05$ & $1.86+/-0.18$ & $11.70+/-0.84$ \\
\hline $22-23$ & 1.18 & 0.49 & 0.58 & 10.88 & $1.47+/-0.15$ & $0.24+/-0.03$ & $1.94+/-0.14$ & $0.69+/-0.04$ & $1.74+/-0.14$ & $12.57+/-0.72$ \\
\hline $23-24$ & 1.11 & 0.46 & 0.58 & 10.33 & $1.39+/-0.08$ & $0.22+/-0.03$ & $2.19+/-0.15$ & $0.66+/-0.04$ & $1.93+/-0.16$ & $13.09+/-0.77$ \\
\hline $23-24^{\mathrm{c}}$ & & & & 10.50 & $1.42+/-0.05$ & & & & & \\
\hline $24-25$ & 1.14 & 0.46 & 0.60 & 9.93 & $1.34+/-0.05$ & $0.17+/-0.03$ & $1.90+/-0.16$ & $0.70+/-0.05$ & $1.82+/-0.18$ & $12.95+/-0.85$ \\
\hline $24-25^{\mathrm{c}}$ & & & & 10.70 & $1.53+/-0.03$ & & & & & \\
\hline $25-26$ & 1.17 & 0.50 & 0.57 & 9.70 & $1.14+/-0.01$ & $0.14+/-0.03$ & $1.58+/-0.14$ & $0.78+/-0.05$ & $1.82+/-0.16$ & $12.83+/-0.75$ \\
\hline $26-27$ & 1.19 & 0.50 & 0.58 & 10.05 & $0.96+/-0.04$ & $0.08+/-0.03$ & $1.40+/-0.16$ & $0.73+/-0.05$ & $1.63+/-0.17$ & $12.00+/-0.83$ \\
\hline $26-27^{\mathrm{c}}$ & & & & 8.54 & $1.02+/-0.01$ & & & & & \\
\hline $27-28$ & 1.18 & 0.51 & 0.57 & 7.91 & $0.82+/-0.02$ & $0.04+/-0.03$ & $1.41+/-0.17$ & $0.79+/-0.05$ & $1.70+/-0.19$ & $12.39+/-0.87$ \\
\hline $28-29$ & 1.12 & 0.49 & 0.56 & 7.62 & $0.66+/-0.08$ & ND & $1.15+/-0.12$ & $0.77+/-0.04$ & $1.61+/-0.15$ & $12.51+/-0.72$ \\
\hline $28-29^{c}$ & & & & 10.08 & $0.77+/-0.01$ & & & & & \\
\hline $29-30$ & 1.19 & 0.52 & 0.56 & 7.80 & $0.65+/-0.07$ & ND & $1.23+/-0.15$ & $0.75+/-0.05$ & $1.44+/-0.17$ & $12.05+/-0.87$ \\
\hline $30-31$ & 1.21 & 0.53 & 0.56 & 7.90 & $0.76+/-0.01$ & ND & $1.08+/-0.15$ & $0.85+/-0.05$ & $1.48+/-0.19$ & $13.28+/-0.89$ \\
\hline $31-32$ & 1.20 & 0.53 & 0.56 & & & & & & & \\
\hline
\end{tabular}

$\mathrm{a}=$ results determined by alpha spectroscopy method; $\mathrm{b}=$ results determined by gamma spectroscopy method; $\mathrm{c}=$ replicate analysis

$\mathrm{L}_{\mathrm{c}: \mathrm{Cs}-137}=0.03 \mathrm{pCi} / \mathrm{g} ; \mathrm{L}_{\mathrm{c}: \mathrm{Pb}-210}=0.11 \mathrm{pCi} / \mathrm{g} ; \mathrm{L}_{\mathrm{c}: \mathrm{Ra}-226}=0.07 \mathrm{pCi} / \mathrm{g} ; \mathrm{L}_{\mathrm{c}: \mathrm{Th}-234}=0.12 \mathrm{pCi} / \mathrm{g} ; \mathrm{L}_{\mathrm{c}: \mathrm{K}-40}=0.41 \mathrm{pCi} / \mathrm{g}$ 